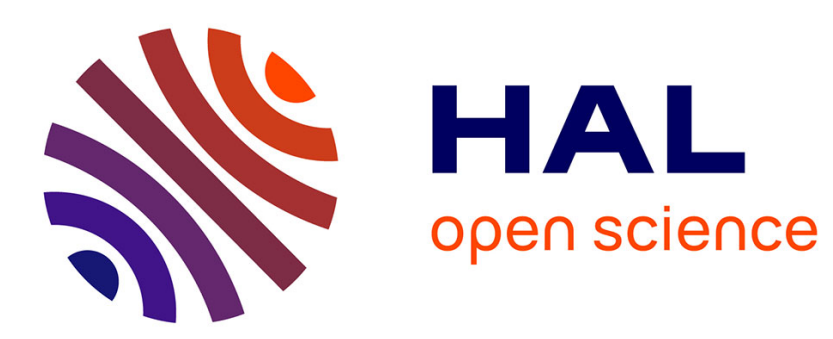

\title{
Comparison of the 3rd order dispersion terms of interaction between chiral molecules
}

Richard Bonneville

\section{To cite this version:}

Richard Bonneville. Comparison of the 3rd order dispersion terms of interaction between chiral molecules. Molecular Physics, 2011, 109 (05), pp.751-759. 10.1080/00268976.2011.552442 . hal00676092

\section{HAL Id: hal-00676092 \\ https://hal.science/hal-00676092}

Submitted on 3 Mar 2012

HAL is a multi-disciplinary open access archive for the deposit and dissemination of scientific research documents, whether they are published or not. The documents may come from teaching and research institutions in France or abroad, or from public or private research centers.
L'archive ouverte pluridisciplinaire HAL, est destinée au dépôt et à la diffusion de documents scientifiques de niveau recherche, publiés ou non, émanant des établissements d'enseignement et de recherche français ou étrangers, des laboratoires publics ou privés. 


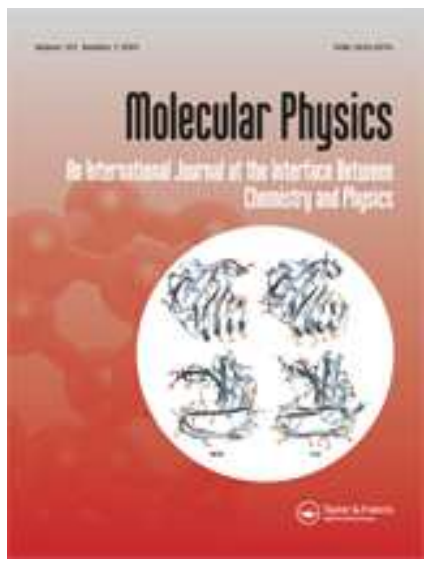

\section{Comparison of the 3rd order dispersion terms of interaction between chiral molecules}

\begin{tabular}{|r|l|}
\hline Journal: & Molecular Physics \\
\hline Manuscript ID: & TMPH-2010-0418.R1 \\
\hline Manuscript Type: & Full Paper \\
\hline $\begin{array}{r}\text { Date Submitted by the } \\
\text { Author: }\end{array}$ & 15-Dec-2010 \\
\hline Complete List of Authors: & BONNEVILLE, Richard; CNES, DSP/DA \\
\hline Keywords: & $\begin{array}{l}\text { chiral molecules, electrodynamics, molecular interactions, non } \\
\text { linear optics }\end{array}$ \\
\hline
\end{tabular}

\section{SCHOLARONE ${ }^{m}$ Manuscripts}




\title{
Comparison of the $3^{\text {rd }}$ order dispersion terms of interaction between chiral molecules
}

\author{
Richard BONNEVILLE \\ CNES, 2 place Maurice Quentin, 75001 Paris, France \\ 33.80.-b, 33.55.Ad, 33.15.-e, 33.00.00, 34.20.Gj, 61.30.-v, 61.30.Gd
}

\begin{abstract}
Through a quantum electrodynamic approach, we investigate the coupling between chiral molecules deriving from the exchange of 3 virtual photons of the electric dipole type. The resulting interaction potential, which involves the first nonlinear polarizability of the molecules, is compared with a previously derived interaction potential involving the optical activity of the molecules; the latter is accounted for by the exchange of 2 virtual photons, one being electric dipolar and the other being electric quadrupolar or magnetic dipolar. The symmetry properties of the new term is considered and its quasi static limit is derived. It appears that the two interaction terms, which both are third-order dispersion processes, might have the same order of magnitude; thus none can a priori be neglected and both might equally contribute to the possibility of an helical ordering.
\end{abstract}




\section{Introduction}

When they are coupled with the electromagnetic field, chiral molecules exhibit two noticeable characteristics: (i) they are optically active, (ii) they have a non-zero first non linear polarizability. The rank-3 true tensor $\eta_{\text {ipj }}(\omega)$ which accounts for optical activity is anti-symmetric in the exchange of its extreme indices $i$ and $j$ from a general property of the kinetic coefficients and thus it is equivalent to a rank-2 pseudo-tensor [1]. The first non linear polarizability responsible for the parametric optical phenomena at molecular level [2] is expressed by rank-3 true tensor $\beta_{\mathrm{ijk}}\left(\omega_{1}, \omega_{2}, \omega_{3}\right)$ with $\omega_{1}+\omega_{2}+\omega_{3}=0$.

In a recent paper [3] we had reviewed the symmetries of the electromagnetic interactions between chiral molecules. Molecular interactions include longitudinal static couplings between permanent dipoles and transverse dynamic couplings; in the quantum electrodynamics formulation of the field - matter interactions the latter are viewed as resulting from an exchange of virtual photons between the molecules.

The lowest order term of those dynamic interactions involves four coupling vortices and the exchange of two photons. If those elementary couplings are of electric dipole type, we obtain the well-known Van der Waals interaction which can be expressed as a function of the linear polarizability $\alpha$ of the two molecules and which varies as $\mathrm{R}^{-6}$ in the short range limit. If we go beyond the electric dipole approximation and consider magnetic dipole or electric quadrupole type couplings, additional interaction terms can be evidenced between chiral molecules, with a $\mathrm{R}^{-8}$ dependence in the short range limit. Those chiral terms can be expressed as a function of the optical activity $\eta$ of the two molecules. 
The association of the anisotropic part of those chiral terms with the anisotropic part of the Van der Waals interaction can account for the helicoidal order of the chiral nematics (cholesteric phases). The anisotropic Van der Waals interaction can be expressed as a function of the scalar product $\alpha^{(2)}(\mathrm{A}) \cdot \alpha^{(2)}(\mathrm{B})$ of the quadrupolar components of the linear polarizabilities, and the anisotropic chiral interaction can be expressed as a function of the scalar product $\eta^{(2)}(\mathrm{A}) \cdot \eta^{(2)}(\mathrm{B})$ of the quadrupolar components of the optical activities. Both interactions have the same rotational symmetry properties, but the Van der Waals term is always attractive and tends to align the molecules parallel or antiparallel to one of the main axes of $\alpha^{(2)}$, conversely the chiral term is always repulsive and tends to align the molecules perpendicular to one of the main axes of $\eta^{(2)}$ (N.B.: the main axes of the molecular tensors $\alpha^{(2)}$ and $\eta^{(2)}$ are a priori different). The 2 terms thus tend to align the molecules along two different directions; it is well known that a system undergoing forces with different symmetries and ranges may minimise its free energy by adopting an helical ordering. The $\mathrm{Z}$ axis of $\alpha^{(2)}$ and the $\mathrm{Z}$ axis of $\eta^{(2)}$ define a molecular plane $\Pi$. The interaction energy between 2 molecules A and B is minimal if either the molecular planes $\Pi_{A}$ and $\Pi_{B}$ are identical, i.e. there is a nematic-type order in that plane, or if $\Pi_{A}$ and $\Pi_{B}$ are parallel, the nematic axes of the 2 planes being shifted by a certain angle, which generates a cholesteric-type twist.

\section{Nonlinear coupling between chiral molecules}


In the quasi-static limit (Appendix 1) the dynamical expression of the Van der Waals forces gives again the classical expression derived from a static dipole-dipole interaction at the $2^{\text {nd }}$ order of perturbation:

$\mathrm{V}_{\mathrm{AB}}=\sum_{\mathrm{m}, \mathrm{n}} \frac{\left\langle 0_{\mathrm{A}} 0_{\mathrm{B}}\left|\mathrm{d}_{\mathrm{A}} \mathrm{T}_{\mathrm{AB}} \mathrm{d}_{\mathrm{B}}\right| \mathrm{m}_{\mathrm{A}} \mathrm{n}_{\mathrm{B}}\right\rangle\left\langle\mathrm{m}_{\mathrm{A}} \mathrm{n}_{\mathrm{B}}\left|\mathrm{d}_{\mathrm{A}} \mathrm{T}_{\mathrm{AB}} \mathrm{d}_{\mathrm{B}}\right| 0_{\mathrm{A}} 0_{\mathrm{B}}\right\rangle}{2 \mathrm{E}_{0}-\mathrm{E}_{\mathrm{m}}-\mathrm{E}_{\mathrm{n}}}$

where $T_{A B}$ is the static dipole-dipole coupling tensor, leading to the R-dependence in $\mathrm{R}^{-6}$. Now it has been pointed out by Schipper [4] that a $3^{\text {rd }}$ order perturbation term of the static dipole-dipole interaction would give a non-zero contribution for chiral molecules, namely:

$\mathrm{V}_{\mathrm{AB}}=\sum_{m, n, m^{\prime}, n^{\prime}} \frac{\left\langle 0_{\mathrm{A}} 0_{B}\left|\mathrm{~d}_{\mathrm{A}} \mathrm{T}_{\mathrm{AB}} \mathrm{d}_{\mathrm{B}}\right| \mathrm{m}_{\mathrm{A}}{ }_{\mathrm{A}} \mathrm{n}^{\prime}{ }_{\mathrm{B}}\right\rangle\left\langle\mathrm{m}^{\prime}{ }_{\mathrm{A}} \mathrm{n}^{\prime}{ }_{\mathrm{B}}\left|\mathrm{d}_{\mathrm{A}} \mathrm{T}_{\mathrm{AB}} \mathrm{d}_{\mathrm{B}}\right| \mathrm{m}_{\mathrm{A}} \mathrm{n}_{\mathrm{B}}\right\rangle\left\langle\mathrm{m}_{\mathrm{A}} \mathrm{n}_{\mathrm{B}}\left|\mathrm{d}_{\mathrm{A}} \mathrm{T}_{\mathrm{AB}} \mathrm{d}_{\mathrm{B}}\right| 0_{\mathrm{A}} 0_{\mathrm{B}}\right\rangle}{\left(2 \mathrm{E}_{0}-\mathrm{E}_{\mathrm{m}^{\prime}}-\mathrm{E}_{\mathrm{n}^{\prime}}\right)\left(2 \mathrm{E}_{0}-\mathrm{E}_{\mathrm{m}}-\mathrm{E}_{\mathrm{n}}\right)}$

with a R-dependence in $\mathrm{R}^{-9}$. Then Harris [5] have shown that this $\mathrm{R}^{-9}$ interaction could be expressed as a double integral over frequencies of a product of 2 complex sum frequency generation responses.

Actually, the expression equ.(2) above is the static limit of a dynamic process in which the calculation of the intermolecular energy expresses the exchange of 3 virtual photons between the two molecules with the 6 coupling vortices being of electric dipole type. More explicitly, 2 types of field-matter couplings can be called for each of the two molecules:

(i) either the absorption of two photons of respective frequencies $\omega$ and $\omega^{\prime}$ and the emission of one photon of frequency $\omega^{\prime \prime}=\omega+\omega^{\prime}$, that is accounted for by 6 terms obtained by permutation of the 3 vortices of fig.(1a), 
(ii) or the emission of two photons of respective frequencies $\omega$ and $\omega^{\prime}$ and the absorption of one photon of frequency $\omega^{\prime \prime}=\omega^{\prime}+\omega$, that is accounted for by 6 terms obtained by permutation of the 3 vortices of fig.(1b).

The nonlinear interaction between molecule A and molecule B is thus accounted for either by process (i) for molecule A associated to process (ii) for molecule B, i.e. 36 terms, or in a symmetric way by process (ii) for molecule A associated to process (i) for molecule B, i.e. 36 terms. Finally, there are 72 terms in total describing the whole process, each belonging to one of the 3 topological types of fig. 2 .

By following the same approach as in ref.[3], the 3-photons transition amplitude after integration over the 6 interaction times between an initial instant $t_{i} \rightarrow-\infty$ when the field - matter coupling is switched on and a final instant $t_{f} \rightarrow+\infty$ where it is switched off can be expressed (Appendix 2) as

$$
\begin{aligned}
\mathrm{T}_{\mathrm{i} \rightarrow \mathrm{f}}= & \mathrm{T}_{\mathrm{i} \rightarrow \mathrm{f}}^{0}+\left( \pm \mathrm{i} \hbar / 4 \pi^{2}\right)\left(\mathrm{t}_{\mathrm{f}}-\mathrm{t}_{\mathrm{i}}\right)\left(4 \pi \epsilon_{0}\right)^{-3} \mathrm{R}^{-9} \exp \left[-\mathrm{iE}_{0}\left(\mathrm{t}_{\mathrm{f}}-\mathrm{t}_{\mathrm{i}}\right) \hbar^{-1}\right] \int_{0}^{+\infty} d \omega \int_{0}^{+\infty} d \omega^{\prime} \\
& \sum_{\mathrm{ijkpqr}}\left(\begin{array}{l}
\beta_{\mathrm{urp}}\left(-\omega^{\prime}-\omega, \omega^{\prime}, \omega\right)_{\mathrm{A}} \beta_{\mathrm{vsq}}\left(\omega^{\prime}+\omega,-\omega^{\prime},-\omega\right)_{\mathrm{B}} \mathrm{D}_{\mathrm{uv}}\left(\mathrm{i}\left(\omega^{\prime}+\omega\right) \mathrm{R} / \mathrm{c}\right) \mathrm{D}_{\mathrm{rs}}\left(\mathrm{i} \omega^{\prime} \mathrm{R} / \mathrm{c}\right) \mathrm{D}_{\mathrm{pq}}(\mathrm{i} \omega \mathrm{R} / \mathrm{c}) \\
+\beta_{\mathrm{urp}}\left(\omega^{\prime}+\omega,-\omega^{\prime},-\omega\right)_{\mathrm{A}} \beta_{\mathrm{vsq}}\left(-\omega^{\prime}-\omega, \omega^{\prime}, \omega\right)_{\mathrm{B}} \mathrm{D}_{\mathrm{uv}}\left(-\mathrm{i}\left(\omega^{\prime}+\omega\right) \mathrm{R} / \mathrm{c}\right) \mathrm{D}_{\mathrm{rs}}\left(-\mathrm{i} \omega^{\prime} \mathrm{R} / \mathrm{c}\right) \mathrm{D}_{\mathrm{pq}}(-\mathrm{i} \omega \mathrm{R} / \mathrm{c})
\end{array}\right.
\end{aligned}
$$

$\mathrm{T}_{\mathrm{i} \rightarrow \mathrm{f}}^{0}=\exp \left[-\mathrm{iE}_{0}\left(\mathrm{t}_{\mathrm{f}}-\mathrm{t}_{\mathrm{i}}\right) \hbar^{-1}\right]$ accounts for the trivial un-perturbed case and $\mathrm{D}_{\mathrm{j} 1}$ is given by

$D_{j 1}(x)=\delta_{j 1}\left(1-x+x^{2}\right) \exp (x)-\frac{R_{j} R_{1}}{R^{2}}\left(3-3 x+x^{2}\right) \exp (x)$

The two nonlinear polarizability tensors $\beta_{\mathrm{ijk}}\left(-\omega^{\prime}-\omega^{\prime} \omega^{\prime}, \omega\right)$ and $\beta_{\mathrm{ijk}}\left(\omega^{\prime}+\omega,-\omega^{\prime},-\omega\right)$ are naturally introduced in a symmetrical way.

Calling $\Delta \mathrm{E}$ the energy shift caused by the molecular interactions so that 


$$
\mathrm{T}_{\mathrm{i} \rightarrow \mathrm{f}}=\exp \left[-\mathrm{i}\left(\mathrm{E}_{0}+\Delta \mathrm{E}\right)\left(\mathrm{t}_{\mathrm{f}}-\mathrm{t}_{\mathrm{i}}\right) \hbar^{-1}\right] \cong\left[1-\mathrm{i} \Delta \mathrm{E}\left(\mathrm{t}_{\mathrm{f}}-\mathrm{t}_{\mathrm{i}}\right) \hbar^{-1}\right] \exp \left[-\mathrm{iE}_{0}\left(\mathrm{t}_{\mathrm{f}}-\mathrm{t}_{\mathrm{i}}\right) \hbar^{-1}\right]
$$

we get by comparing equ.(3) and equ.(5)

$$
\begin{aligned}
\Delta \mathrm{E}= & \operatorname{Re}\left(\hbar^{2} / 4 \pi^{2}\right)\left(4 \pi \epsilon_{0}\right)^{-3} \mathrm{R}^{-9} \int_{0}^{+\infty} d \omega \int_{0}^{+\infty} d \omega^{\prime} \\
& \sum_{\mathrm{ijkpqr}}\left(\begin{array}{l}
\beta_{\mathrm{urp}}\left(-\omega^{\prime}-\omega, \omega^{\prime}, \omega\right)_{\mathrm{A}} \beta_{\mathrm{vsq}}\left(\omega^{\prime}+\omega,-\omega^{\prime},-\omega\right)_{\mathrm{B}} \mathrm{D}_{\mathrm{uv}}\left(\mathrm{i}\left(\omega^{\prime}+\omega\right) \mathrm{R} / \mathrm{c}\right) \mathrm{D}_{\mathrm{rs}}\left(\mathrm{i} \omega^{\prime} \mathrm{R} / \mathrm{c}\right) \mathrm{D}_{\mathrm{pq}}(\mathrm{i} \omega \mathrm{R} / \mathrm{c}) \\
+\beta_{\mathrm{urp}}\left(\omega^{\prime}+\omega,-\omega^{\prime},-\omega\right)_{\mathrm{A}} \beta_{\mathrm{vsq}}\left(-\omega^{\prime}-\omega, \omega^{\prime}, \omega\right)_{\mathrm{B}} \mathrm{D}_{\mathrm{uv}}\left(-\mathrm{i}\left(\omega^{\prime}+\omega\right) \mathrm{R} / \mathrm{c}\right) \mathrm{D}_{\mathrm{rs}}\left(-\mathrm{i} \omega^{\prime} \mathrm{R} / \mathrm{c}\right) \mathrm{D}_{\mathrm{pq}}(-\mathrm{i} \omega \mathrm{R} / \mathrm{c})
\end{array}\right.
\end{aligned}
$$

The interaction energy is thus expressed as a double integral over frequencies of a product of the non linear polarizabilities of the two molecules. As far as the Rdependence is concerned, the leading term in equ.(6) decreases with distance as $\mathrm{R}^{-9}$ as expected.

$\mathrm{D}_{\mathrm{jl}}$ can be written as

$D_{j 1}(x)=\delta_{j 1} \frac{2}{3} x^{2} \exp (x)-\left(\frac{3 R_{j} R_{1}-R^{2} \delta_{j 1}}{2 R^{2}}\right) \frac{2}{3}\left(3-3 x+x^{2}\right) \exp (x)$

As a function of $\mathbf{R} \mathrm{D}_{\text {ip }}$ is the sum of two totally symmetric (with respect to index permutations) tensors irreducible under the rotation group, a scalar one $(\mathrm{J}=0)$ and a quadrupolar one $(\mathrm{J}=2)$. Consequently $\mathrm{D}_{\mathrm{ip}} \mathrm{D}_{\mathrm{jq}} \mathrm{D}_{\mathrm{kr}}$ is the sum of terms of weight $\mathrm{J}=0, \mathrm{~J}=2$, $\mathrm{J}=4$ and $\mathrm{J}=6$, among which, assuming an isotropic distribution of the molecular centres, we will only retain the scalar contribution $(\mathrm{J}=0)$.

In terms of irreducible tensor components with respect to rotations and index permutations, $\beta_{\mathrm{ijk}}$ has the following spectrum $[3,6]$ :

$\{3\}\left(\beta^{(3)}+\beta^{(1)}\right),\{21\}\left(\beta^{\prime(2)}+\beta^{\prime \prime(2)}+\beta^{\prime(1)}+\beta^{\prime \prime(1)}\right),\left\{1^{3}\right\} \beta^{(0)}$ 
Assuming an isotropic distribution of the molecular centres, the intermolecular coupling has thus the following form:

$$
\begin{aligned}
\Delta \mathrm{E} \propto & \int_{0}^{+\infty} \int_{0}^{+\infty} d \omega d \omega^{\prime} \mathrm{R}^{-9} \Phi\left(\omega \mathrm{R} / \mathrm{c}, \omega^{\prime} \mathrm{R} / \mathrm{c}\right) \\
& \left(\begin{array}{c}
\left.\sum_{\mathrm{J}=0,1,2,3} \begin{array}{r}
\beta^{(\mathrm{J})}\left(-\omega^{\prime}-\omega, \omega^{\prime}, \omega\right)_{\mathrm{B}} \cdot \beta^{(\mathrm{J})}\left(\omega^{\prime}+\omega,-\omega^{\prime},-\omega\right)_{\mathrm{A}}+ \\
\beta^{(\mathrm{J})}\left(\omega^{\prime}+\omega,-\omega^{\prime},-\omega\right)_{\mathrm{B}} \cdot \beta^{(\mathrm{J})}\left(-\omega^{\prime}-\omega, \omega^{\prime}, \omega\right)_{\mathrm{A}}
\end{array}\right)
\end{array}\right.
\end{aligned}
$$

\section{Quasi static limit}

In the Miller approximation for $\beta_{\mathrm{ijk}}$ where the frequency dispersion is neglected [2], the remnant terms are the octupole-type and vector-type components $\{3\} \beta^{(3)}$ and $\{3\} \beta^{(1)}$, fully symmetric with respect to any index permutation. $\Delta \mathrm{E}$ is then essentially the sum of a vector-vector coupling and of an octupole-octupole coupling, which have to be added to the Van der Waals forces and to the chiral forces associated to optical activity.

If the retardation effects are neglected $(\omega \mathrm{R} / \mathrm{c} \ll 1)$, then

$\mathrm{D}_{\text {ip }} \rightarrow \delta_{\text {ip }}-\frac{3 \mathrm{R}_{\mathrm{i}} \mathrm{R}_{\mathrm{p}}}{\mathrm{R}^{2}}$

i.e. $\mathrm{D}_{\mathrm{ip}}$ as a function of $\mathbf{R}$ is reduced to a pure quadrupole, independent of the frequency.

In the quasi static limit, the scalar (i.e. isotropic) contribution of $\mathrm{D}_{\mathrm{ip}} \mathrm{D}_{\mathrm{jq}} \mathrm{D}_{\mathrm{kr}}$ can be checked to be equal to

$\mathrm{D}_{\mathrm{ip}} \mathrm{D}_{\mathrm{jq}} \mathrm{D}_{\mathrm{kr}} \rightarrow-\frac{16}{35} \delta_{\mathrm{ip}} \delta_{\mathrm{jq}} \delta_{\mathrm{kr}}$

so that 


$$
\Delta \mathrm{E}=\frac{1}{2 \pi} \frac{16}{35} \frac{1}{\mathrm{R}^{-9}} \operatorname{Re} \int_{0}^{+\infty} d \omega \int_{0}^{+\infty} d \omega^{\prime} \sum_{\mathrm{ijk}}\left(\begin{array}{c}
\beta_{\mathrm{ijk}}\left(-\omega^{\prime}-\omega, \omega^{\prime}, \omega\right)_{\mathrm{B}} \beta_{\mathrm{ijk}}\left(\omega^{\prime}+\omega,-\omega^{\prime},-\omega\right)_{\mathrm{A}}+ \\
\beta_{\mathrm{ijk}}\left(\omega^{\prime}+\omega,-\omega^{\prime},-\omega\right)_{\mathrm{B}} \beta_{\mathrm{ijk}}\left(-\omega^{\prime}-\omega, \omega^{\prime}, \omega\right)_{\mathrm{A}}
\end{array}\right)
$$

The integrals over the frequencies can be easily calculated by using

$$
\frac{1}{\omega-\omega_{0} \pm \mathrm{i} \varepsilon}=\mathrm{PP} \frac{1}{\omega-\omega_{0}} \mp \mathrm{i} \pi \delta\left(\omega-\omega_{0}\right)
$$

It appears that the real and imaginary contributions of most of the 72 terms cancel each other 2 by 2 ; actually only 4 terms described by the diagrams of fig. 3 have a non vanishing contribution (Appendix 3).

Finally we have

$$
\Delta E=\frac{16}{35} \frac{1}{R^{9}} \frac{1}{\left(4 \pi \epsilon_{0}\right)^{3}} \sum_{\mathrm{ijk}} \sum_{m, n} \sum_{m^{\prime}, n^{\prime}} \frac{\left\langle 0\left|\mathrm{~d}_{\mathrm{i}} \mathrm{P}_{\mathrm{m}^{\prime}} \mathrm{d}_{\mathrm{j}} \mathrm{P}_{\mathrm{m}} \mathrm{d}_{\mathrm{k}}\right| 0\right\rangle\left\langle 0\left|\mathrm{~d}_{\mathrm{i}} \mathrm{P}_{\mathrm{n}^{\prime}} \mathrm{d}_{\mathrm{j}} \mathrm{P}_{\mathrm{n}} \mathrm{d}_{\mathrm{k}}\right| 0\right\rangle}{\left(2 \mathrm{E}_{0}-\mathrm{E}_{\mathrm{m}^{\prime}}-\mathrm{E}_{\mathrm{n}^{\prime}}\right)\left(2 \mathrm{E}_{0}-\mathrm{E}_{\mathrm{m}}-\mathrm{E}_{\mathrm{n}}\right)}
$$

It is easily verified that the resulting expression is the static term proposed in equ.(2) after averaging over molecular orientations. That interaction is always attractive and tends to align the molecules.

\section{Possibility of an helicoidal ordering}

$\beta^{(1)}$ and $\beta^{(3)}$ behave in a rotation like the irreducible unit tensors [7] $\mathrm{C}^{(1)}(\theta, \varphi)$ and $\mathrm{C}^{(3)}(\theta, \varphi)$ whereas $\alpha^{(2)}$ and $\eta^{(2)}$ behave like $\mathrm{C}^{(2)}(\theta, \varphi)$. Let $\theta_{\mathrm{A}}, \varphi_{\mathrm{A}}\left(\operatorname{resp} \theta_{\mathrm{B}}, \varphi_{\mathrm{B}}\right)$ be the polar angles of a molecular axis $\mathrm{Z}_{\mathrm{A}}\left(\right.$ resp. $\mathrm{Z}_{\mathrm{B}}$ ) in some external reference frame and let $\rho_{\mathrm{AB}}$ be the angle between $Z_{A}$ and $Z_{B}$. The interaction energy between the 2 molecules $A$ and $\mathrm{B}$ can be written as

$\mathrm{U}_{\mathrm{AB}}=\mathcal{E}_{1}(\mathrm{R}) \mathrm{P}_{1}\left(\cos \rho_{\mathrm{AB}}\right)+\mathcal{E}_{2}(\mathrm{R}) \mathrm{P}_{2}\left(\cos \rho_{\mathrm{AB}}\right)+\mathcal{E}_{3}(\mathrm{R}) \mathrm{P}_{3}\left(\cos \rho_{\mathrm{AB}}\right)$

$\mathrm{P}_{\ell}\left(\cos \rho_{\mathrm{AB}}\right)$ is the Legendre polynomial of order $\ell$ 
with $\mathrm{P}_{\ell}\left(\cos \rho_{\mathrm{AB}}\right)=\mathrm{C}^{(\ell)}\left(\theta_{\mathrm{A}}, \varphi_{\mathrm{A}}\right) \cdot \mathrm{C}^{(\ell)}\left(\theta_{\mathrm{B}}, \varphi_{\mathrm{B}}\right)$

and $\cos \rho_{\mathrm{AB}}=\cos \theta_{\mathrm{A}} \cos \theta_{\mathrm{B}}+\sin \theta_{\mathrm{A}} \sin \theta_{\mathrm{B}} \cos \left(\varphi_{\mathrm{A}}-\varphi_{\mathrm{B}}\right)$

It is worth recalling here that in the situation considered in ref.[3] the helicoidal ordering derives from the conflict between the quadrupolar Van der Waals interaction and the quadrupolar chiral interaction. Both interactions have the same symmetry properties with respect to rotations, and different ranges, but the Van der Waals term is always attractive and tends to align the molecules parallel or anti-parallel to one of the main axes of $\alpha^{(2)}$, conversely the chiral term is always repulsive and tends to align the molecules perpendicular to one of the main axes of $\eta^{(2)}$. The 2 couplings thus tend to align the molecules along two different directions and the system may minimise its free energy by adopting an helical ordering.

In the present situation, the molecular coupling associated with the nonlinear polarizabilities has a different symmetry property with respect to rotations (and also a different range) and that conflict could also cause an helicoidal ordering (the situation is somehow similar to the helimagnetic case).

The molecular planes $\Pi_{\mathrm{A}}$ and $\Pi_{\mathrm{B}}$ intersect each other along a direction which we choose as the $\mathrm{Z}$ axis. Let $\Phi$ be the angle between $\Pi_{\mathrm{A}}$ and $\Pi_{\mathrm{B}}$ (fig. 4) so that $\Phi=\varphi_{A}-\varphi_{B}$. Writing $\left(\frac{\partial U}{\partial \Phi}\right)_{R}=0$, the minima of $U$ for any given value of $R$ are the roots of $\sin \rho_{A B}=0$ i.e. $\rho_{A B}=0$ or $\rho_{A B}=\pi ; \rho_{A B}=0$ means $\Phi=0, \theta_{\mathrm{A}}=\theta_{\mathrm{B}}$, i.e. a parallel configuration, and it corresponds to a minimum of $\mathrm{U}$ whereas $\rho_{A B}=\pi$ means 
$\Phi=\pi, \theta_{\mathrm{B}}=-\theta_{\mathrm{A}}$ and corresponds to a maximum of $\mathrm{U}$. In the parallel configuration, $\Pi_{\mathrm{A}}$ and $\Pi_{\mathrm{B}}$ are identical and in that plane the molecules present a nematic-type ordering. If $\Pi_{\mathrm{A}}$ and $\Pi_{\mathrm{B}}$ are parallel, the $\mathrm{Z}$ axis as chosen above (intersection of $\Pi_{\mathrm{A}}$ and $\Pi_{\mathrm{B}}$ ) does not exist and we adopt a different approach. Let $\mathrm{Z}$ now be the axis perpendicular to $\Pi_{\mathrm{A}}$ and $\Pi_{\mathrm{B}}$ and let $\Theta$ be the angle between $Z$ and $\mathbf{R}$ (fig. 5).

We look for the possibility of helical ordering by putting $\mathrm{t}=\cos \rho_{\mathrm{AB}}, \rho_{\mathrm{AB}}=\mathrm{qZ}, \mathrm{Z}=\mathrm{R} \cos \Theta$

At short distance, the molecules feel a strong repulsive interaction due to the exclusion principle; as they are supposed to be able to rotate freely and as their centres are assumed to be randomly distributed, the repulsive forces will be accounted for by an effective hard-core inter-molecular potential of radius D (effective molecular diameter).

We will express the quadrupole-type interaction as $\mathcal{E}_{2}(\mathrm{R})=\left\langle\mathcal{E}_{\mathrm{VdW}}\right\rangle\left(\frac{\mathrm{D}}{\mathrm{R}}\right)^{6}-\left\langle\mathcal{E}_{\eta}\right\rangle\left(\frac{\mathrm{D}}{\mathrm{R}}\right)^{8}$ for $\mathrm{R}>\mathrm{D}$,

where the first term accounts for the anisotropic Van der Waals interaction and the second term for the interaction associated to the optical activity, the vector-type coupling associated to the nonlinear polarizability as $\mathcal{E}_{1}(\mathrm{R})=\left\langle\mathcal{E}_{1}\right\rangle\left(\frac{\mathrm{D}}{\mathrm{R}}\right)^{9}$ for $\mathrm{R}>\mathrm{D}$, and the octupole-type coupling associated to the nonlinear polarizability as $\mathcal{E}_{3}(\mathrm{R})=\left\langle\mathcal{E}_{3}\right\rangle\left(\frac{\mathrm{D}}{\mathrm{R}}\right)^{9}$ for $\mathrm{R}>\mathrm{D}$

The average interaction energy of 2 molecules A and B can thus be written as 


$$
\begin{aligned}
\left\langle\mathrm{U}_{\mathrm{AB}}\right\rangle= & \int \frac{d^{3} \mathbf{R}_{\mathrm{AB}}}{\mathrm{V}} \mathrm{Y}(\mathrm{R}-\mathrm{D}) \mathrm{U}_{\mathrm{AB}}(\mathrm{R}) \\
& =\left\langle-\mathrm{H}_{0}\right\rangle+\int \frac{\mathrm{R}^{2} d \mathrm{R}}{\mathrm{V}} \mathrm{Y}(\mathrm{R}-\mathrm{D})\left[\left\langle\mathcal{E}_{\mathrm{VdW}}\right\rangle\left(\frac{\mathrm{D}}{\mathrm{R}}\right)^{6}-\left\langle\mathcal{E}_{\eta}\right\rangle\left(\frac{\mathrm{D}}{\mathrm{R}}\right)^{8}\right] \int 2 \pi \sin \Theta d \Theta \mathrm{P}_{2}\left(\cos \rho_{\mathrm{AB}}\right) \\
& +\int \frac{\mathrm{R}^{2} d \mathrm{R}}{\mathrm{V}} \mathrm{Y}(\mathrm{R}-\mathrm{D})\left[\left\langle\mathcal{E}_{1}\right\rangle\left(\frac{\mathrm{D}}{\mathrm{R}}\right)^{9}\right] \int 2 \pi \sin \Theta d \Theta \mathrm{P}_{1}\left(\cos \rho_{\mathrm{AB}}\right) \\
& +\int \frac{\mathrm{R}^{2} d \mathrm{R}}{\mathrm{V}} \mathrm{Y}(\mathrm{R}-\mathrm{D})+\left[\left\langle\mathcal{E}_{3}\right\rangle\left(\frac{\mathrm{D}}{\mathrm{R}}\right)^{9}\right] \int 2 \pi \sin \Theta d \Theta \mathrm{P}_{3}\left(\cos \rho_{\mathrm{AB}}\right)
\end{aligned}
$$

where $\mathrm{Y}(\mathrm{R}-\mathrm{D})=0$ if $\mathrm{R}<\mathrm{D}$ and $\mathrm{Y}(\mathrm{R}-\mathrm{D})=1$ if $\mathrm{R}>\mathrm{D}$. Writing

$$
\begin{aligned}
& \mathrm{P}_{1}\left(\cos \rho_{\mathrm{AB}}\right)=\cos \rho_{\mathrm{AB}}=\operatorname{Re}(\exp i q \mathrm{R} \cos \Theta) \\
& \mathrm{P}_{2}\left(\cos \rho_{\mathrm{AB}}\right)=\frac{3 \cos ^{2} \rho_{\mathrm{AB}}-1}{2}=\frac{1}{4}+\frac{3}{4} \cos 2 \rho_{\mathrm{AB}}=\frac{1}{4}+\frac{3}{4} \operatorname{Re}(\exp 2 \mathrm{iqR} \cos \Theta) \\
& \mathrm{P}_{3}\left(\cos \rho_{\mathrm{AB}}\right)=\frac{5 \cos \rho_{\mathrm{AB}}{ }^{3}-3 \cos \rho_{\mathrm{AB}}}{2}=\frac{5}{8} \cos 3 \rho_{\mathrm{AB}}+\frac{3}{8} \cos \rho_{\mathrm{AB}} \\
& =\frac{5}{8} \operatorname{Re}(\exp 3 i q \mathrm{R} \cos \Theta)+\frac{3}{8} \operatorname{Re}(\operatorname{expiqR} \cos \Theta)
\end{aligned}
$$

we have

$$
\begin{aligned}
\left\langle\mathrm{U}_{\mathrm{AB}}\right\rangle= & \left\langle-\mathrm{H}_{0}\right\rangle+\int_{\mathrm{D}}^{\infty} \frac{4 \pi \mathrm{R}^{2} d \mathrm{R}}{\mathrm{V}}\left[\left\langle\mathcal{E}_{\mathrm{VdW}}\right\rangle\left(\frac{\mathrm{D}}{\mathrm{R}}\right)^{6}-\left\langle\mathcal{E}_{\mathrm{\eta}}\right\rangle\left(\frac{\mathrm{D}}{\mathrm{R}}\right)^{8}\right]\left[\frac{1}{4}+\frac{3}{4} \frac{\sin 2 \mathrm{qR}}{2 \mathrm{qR}}\right] \\
& +\int_{\mathrm{D}}^{\infty} \frac{4 \pi \mathrm{R}^{2} d \mathrm{R}}{\mathrm{V}}\left[\left\langle\mathcal{E}_{1}\right\rangle\left(\frac{\mathrm{D}}{\mathrm{R}}\right)^{9}\right]\left[\frac{\sin \mathrm{qR}}{\mathrm{qR}}\right] \\
& +\int_{\mathrm{D}}^{\infty} \frac{4 \pi \mathrm{R}^{2} d \mathrm{R}}{\mathrm{V}}\left[\left\langle\mathcal{E}_{3}\right\rangle\left(\frac{\mathrm{D}}{\mathrm{R}}\right)^{9}\right]\left[\frac{5}{8} \frac{\sin 3 \mathrm{qR}}{3 \mathrm{qR}}+\frac{3}{8} \frac{\sin \mathrm{qR}}{\mathrm{qR}}\right]
\end{aligned}
$$

The minimum of $\left\langle\mathrm{U}_{\mathrm{AB}}\right\rangle$ is obtained from $\frac{\partial\left\langle\mathrm{U}_{\mathrm{AB}}\right\rangle}{\partial \mathrm{q}}=0$, i.e. 


$$
\begin{aligned}
0=\frac{3}{2}\left\langle\mathcal{E}_{\mathrm{VdW}}\right\rangle \int_{\mathrm{D}}^{\infty} \frac{d \mathrm{R}}{\mathrm{R}^{3}}\left[\frac{2 \mathrm{qR} \cos 2 \mathrm{qR}-\sin 2 \mathrm{qR}}{(2 \mathrm{qR})^{2}}\right]-\frac{3}{2}\left\langle\mathcal{E}_{\mathrm{\eta}}\right\rangle \mathrm{D}^{2} \int_{\mathrm{D}}^{\infty} \frac{d \mathrm{R}}{\mathrm{R}^{5}}\left[\frac{2 \mathrm{qR} \cos 2 \mathrm{qR}-\sin 2 \mathrm{qR}}{(2 \mathrm{qR})^{2}}\right] \\
+\left[\left\langle\mathcal{E}_{1}\right\rangle+\frac{3}{8}\left\langle\mathcal{E}_{3}\right\rangle\right] \mathrm{D}^{3} \int_{\mathrm{D}}^{\infty} \frac{d \mathrm{R}}{\mathrm{R}^{6}}\left[\frac{\mathrm{qR} \cos \mathrm{qR}-\sin \mathrm{qR}}{(\mathrm{qR})^{2}}\right]+\frac{15}{8}\left\langle\mathcal{E}_{3}\right\rangle \mathrm{D}^{3} \int_{\mathrm{D}}^{\infty} \frac{d \mathrm{R}}{\mathrm{R}^{6}}\left[\frac{3 \mathrm{qR} \cos 3 \mathrm{qR}-\sin 3 \mathrm{qR}}{(3 \mathrm{qR})^{2}}\right]
\end{aligned}
$$

or

$$
\begin{aligned}
0=6\left\langle\mathcal{E}_{\mathrm{VdW}}\right\rangle \int_{2 \mathrm{qD}}^{\infty} \mathrm{dt}\left[\frac{\mathrm{t} \cos \mathrm{t}-\sin \mathrm{t}}{\mathrm{t}^{5}}\right]-24\left\langle\mathcal{E}_{\eta}\right\rangle(\mathrm{qD})^{2} \int_{2 \mathrm{qD}}^{\infty} \mathrm{dt}\left[\frac{\mathrm{t} \cos \mathrm{t}-\sin \mathrm{t}}{\mathrm{t}^{7}}\right] \\
+\left[\left\langle\mathcal{E}_{1}\right\rangle+\frac{3}{8}\left\langle\mathcal{E}_{3}\right\rangle\right](\mathrm{qD})^{3} \int_{\mathrm{qD}}^{\infty} \mathrm{dt}\left[\frac{\mathrm{t} \cos \mathrm{t}-\sin \mathrm{t}}{\mathrm{t}^{8}}\right]+\frac{5.3^{6}}{8}\left\langle\mathcal{E}_{3}\right\rangle(\mathrm{qD})^{3} \int_{3 \mathrm{qD}}^{\infty} \mathrm{dt}\left[\frac{\mathrm{t} \cos \mathrm{t}-\sin \mathrm{t}}{\mathrm{t}^{8}}\right]
\end{aligned}
$$

That allows to determine $\mathrm{q}$ and hence the pitch $\mathrm{L}$ of the helix as $\mathrm{L}=2 \pi / \mathrm{q}$. Assuming

$\mathrm{L}>>\mathrm{D}$, i.e. $\mathrm{qD}<<1$ we get

$$
\begin{aligned}
0=\left\langle\mathcal{E}_{\mathrm{VdW}}\right\rangle & \left(-\frac{1}{\mathrm{qD}}+\frac{3 \pi}{8}+\mathrm{o}(\mathrm{qD})\right)-\frac{1}{3}\left\langle\mathcal{E}_{\mathrm{\eta}}\right\rangle\left(-\frac{1}{\mathrm{qD}}+\mathrm{o}(\mathrm{qD})\right) \\
+ & \frac{1}{12}\left[\left\langle\mathcal{E}_{1}\right\rangle+\frac{3}{8}\left\langle\mathcal{E}_{3}\right\rangle\right]\left(-\frac{1}{\mathrm{qD}}+\mathrm{o}(\mathrm{qD})\right)+\frac{15}{32}\left\langle\mathcal{E}_{3}\right\rangle\left(-\frac{1}{\mathrm{qD}}+\mathrm{o}(\mathrm{qD})\right)
\end{aligned}
$$

and hence

$$
\mathrm{L} \simeq \frac{3 \pi^{2}}{4} \mathrm{D} \frac{\left\langle\mathcal{E}_{\mathrm{VdW}}\right\rangle}{\left\langle\mathcal{E}_{\mathrm{VdW}}\right\rangle-\frac{1}{3}\left\langle\mathcal{E}_{\eta}\right\rangle+\frac{1}{12}\left\langle\mathcal{E}_{1}\right\rangle+\frac{1}{2}\left\langle\mathcal{E}_{3}\right\rangle}
$$

That pitch does not depend upon the temperature as we have performed a simple geometrical averaging; a thermodynamical averaging would have provided a temperature-dependent pitch.

\section{Discussion}


In terms of orders of magnitude, assuming an effective electric charge $\zeta e$ per molecule and an average gap $\hbar \Omega$, we have

$$
\begin{aligned}
& \alpha^{(2)} \sim \frac{(\zeta e)^{2}\left\langle 3 z^{2}-r^{2}\right\rangle}{\hbar \Omega} \\
& \eta^{(2)} \sim \frac{(\zeta e)^{2}\left\langle 6 z^{3}-3 x^{3}-3 y^{3}-r^{2}(2 z-x-y)\right\rangle}{\hbar \Omega} \\
& \beta^{(1)} \sim \frac{(\zeta e)^{3}\left\langle z r^{2}\right\rangle}{(\hbar \Omega)^{2}} \\
& \beta^{(3)} \sim \frac{(\zeta e)^{3}\left\langle 5 z^{3}-3 z\right\rangle}{(\hbar \Omega)^{2}}
\end{aligned}
$$

$\mathcal{E}_{1}$ and $\mathcal{E}_{3}$ are given by an integral over 2 frequencies, $\mathcal{E}_{V d W}$ and $\mathcal{E}_{\eta}$ by an integral over a single frequency. $\Delta / \hbar$ being an effective integration range for the frequencies, we have the following orders of magnitude

$$
\begin{aligned}
& \mathcal{E}_{1} \sim\left(\frac{\zeta^{2} \mathrm{e}^{2}}{4 \pi \epsilon_{0}}\right)^{3}\left(\frac{\left\langle\mathrm{r}^{3}\right\rangle}{\mathrm{R}^{3}}\right)^{2}\left(\frac{\Delta}{\hbar \Omega}\right)^{2}\left(\frac{1}{\hbar \Omega}\right)^{2} \frac{1}{\mathrm{R}^{3}} \\
& \mathcal{E}_{3} \sim\left(\frac{\zeta^{2} \mathrm{e}^{2}}{4 \pi \epsilon_{0}}\right)^{3}\left(\frac{\left\langle\mathrm{r}^{3}\right\rangle}{\mathrm{R}^{3}}\right)^{2}\left(\frac{\Delta}{\hbar \Omega}\right)^{2}\left(\frac{1}{\hbar \Omega}\right)^{2} \frac{1}{\mathrm{R}^{3}} \\
& \mathcal{E}_{\mathrm{VdW}} \sim\left(\frac{\zeta^{2} \mathrm{e}^{2}}{4 \pi \epsilon_{0}}\right)^{2}\left(\frac{\left\langle\mathrm{r}^{2}\right\rangle}{\mathrm{R}^{2}}\right)^{2}\left(\frac{\Delta}{\hbar \Omega}\right) \frac{1}{\hbar \Omega} \frac{1}{\mathrm{R}^{2}} \\
& \mathcal{E}_{\eta} \sim\left(\frac{\zeta^{2} \mathrm{e}^{2}}{4 \pi \epsilon_{0}}\right)^{2}\left(\frac{\left\langle\mathrm{r}^{3}\right\rangle}{\mathrm{R}^{3}}\right)^{2}\left(\frac{\Delta}{\hbar \Omega}\right) \frac{1}{\hbar \Omega} \frac{1}{\mathrm{R}^{2}}
\end{aligned}
$$

and consequently the ratios 


$$
\frac{\mathcal{E}_{\eta}}{\mathcal{E}_{V d W}} \sim\left(\frac{\left\langle r^{3}\right\rangle}{\mathrm{R}\left\langle r^{2}\right\rangle}\right)^{2}
$$

$$
\frac{\mathcal{E}_{1}}{\mathcal{E}_{V d W}} \sim \frac{\mathcal{E}_{3}}{\mathcal{E}_{V d W}} \sim\left(\frac{\zeta^{2} e^{2}}{4 \pi \epsilon_{0}}\right) \frac{1}{\mathrm{R}}\left(\frac{\Delta}{\hbar \Omega}\right) \frac{1}{\hbar \Omega}\left(\frac{\left\langle r^{3}\right\rangle}{\mathrm{R}\left\langle r^{2}\right\rangle}\right)^{2}
$$

$\frac{\mathcal{E}_{1}}{\mathcal{E}_{\eta}} \sim \frac{\mathcal{E}_{3}}{\mathcal{E}_{\eta}} \sim\left(\frac{\zeta^{2} e^{2}}{4 \pi \epsilon_{0}}\right) \frac{1}{\mathrm{R}}\left(\frac{\Delta}{\hbar \Omega}\right) \frac{1}{\hbar \Omega}$

For nearest neighbour molecules $\mathrm{R} \sim \mathrm{D}, \mathrm{D} \sim 10^{-9} \mathrm{~m}, \hbar \Omega \sim \Delta \sim 1-10 \mathrm{eV}$. With those values, it appears that the 3 types of coupling a priori may have the same order of magnitude and hence contribute to the ordering at comparable levels. If however we assume that the Van der Waals coupling is larger than the two chiral couplings, i.e. if the factor $\left(\frac{\left\langle r^{3}\right\rangle}{\mathrm{R}\left\langle r^{2}\right\rangle}\right)^{2}$ is actually small, those chiral couplings have similar magnitudes; thus none of them can be neglected and both contribute at a comparable level to a possible helical ordering. Such an helical ordering is actually observed in the twisted nematics (cholesteric) phases, but further study would be needed in order to precisely attribute their behaviour to the interactions we have highlighted.

\section{Conclusion}

By going beyond the Van der Waals interaction, we have evidenced two different interactions specific to chiral molecules. One can be accounted for by the exchange of 3 virtual photons of the electric dipole type; the resulting interaction potential involves the product of the first nonlinear polarizabilities of the molecules. The other one can be accounted for by the exchange of 2 virtual photons, one being electric dipolar and the 
other being electric quadrupolar or magnetic dipolar; the resulting interaction potential involves the product of the optical activities of the molecules. Both have in common to be third-order dispersion forces. Since they might have the same order of magnitude none can $a$ priori be neglected and both might equally contribute to the possibility of an helical ordering. 


\section{Annexe 1}

In the Van der Waals interaction involving the exchange of 2 photons and 4 electricdipole couplings, the transition amplitude is given by

$$
\begin{aligned}
\mathrm{T}_{\mathrm{i} \rightarrow \mathrm{f}}=\mathrm{T}_{\mathrm{i} \rightarrow \mathrm{f}}^{0}+(2 \pi)^{-1}\left(\mathrm{t}_{\mathrm{f}}-\mathrm{t}_{\mathrm{i}}\right) \exp \left[-\mathrm{iE}_{0}\left(\mathrm{t}_{\mathrm{f}}-\mathrm{t}_{\mathrm{i}}\right) \hbar^{-1}\right] \\
\int_{0}^{+\infty} \mathrm{d} \omega \sum_{\mathrm{ijpq}} \alpha_{\mathrm{ij}}(\omega)_{\mathrm{B}} \alpha_{\mathrm{pq}}(\omega)_{\mathrm{A}} \mathrm{R}^{-6} \mathrm{D}_{\mathrm{ip}}(\mathrm{i} \omega \mathrm{R} / \mathrm{c}) \mathrm{D}_{\mathrm{jq}}(\mathrm{i} \omega \mathrm{R} / \mathrm{c})
\end{aligned}
$$

with

$D_{j 1}(x)=\delta_{j 1}\left(1-x+x^{2}\right) \exp (x)-\frac{R_{j} R_{1}}{R^{2}}\left(3-3 x+x^{2}\right) \exp (x)$

Calling $\Delta \mathrm{E}$ the energy shift caused by the molecular interactions

$$
\mathrm{T}_{\mathrm{i} \rightarrow \mathrm{f}}=\exp \left[-\mathrm{i}\left(\mathrm{E}_{0}+\Delta \mathrm{E}\right)\left(\mathrm{t}_{\mathrm{f}}-\mathrm{t}_{\mathrm{i}}\right) \hbar^{-1}\right] \cong\left[1-\mathrm{i} \Delta \mathrm{E}\left(\mathrm{t}_{\mathrm{f}}-\mathrm{t}_{\mathrm{i}}\right) \hbar^{-1}\right] \exp \left[-\mathrm{iE}_{0}\left(\mathrm{t}_{\mathrm{f}}-\mathrm{t}_{\mathrm{i}}\right) \hbar^{-1}\right]
$$

we get

$$
\Delta \mathrm{E}=\frac{-1}{2 \pi} \operatorname{Im}\left(\int_{0}^{+\infty} \hbar d \omega \sum_{\mathrm{j} p \mathrm{pq}} \alpha_{\mathrm{ij}}(\omega)_{\mathrm{B}} \alpha_{\mathrm{pq}}(\omega)_{\mathrm{A}} \mathrm{R}^{-6} \mathrm{D}_{\mathrm{ip}}(\mathrm{i} \omega \mathrm{R} / \mathrm{c}) \mathrm{D}_{\mathrm{jq}}(\mathrm{i} \omega \mathrm{R} / \mathrm{c})\right)
$$

If the retardation effects are neglected $(\omega \mathrm{R} / \mathrm{c} \ll 1)$, then

$$
\mathrm{D}_{\text {ip }} \rightarrow \mathrm{T}_{\mathrm{ip}}=\delta_{\mathrm{ip}}-\frac{3 \mathrm{R}_{\mathrm{i}} \mathrm{R}_{\mathrm{p}}}{\mathrm{R}^{2}}
$$

i.e. $\mathrm{D}_{\text {ip }}$ as a function of $\mathbf{R}$ is reduced to a pure quadrupole, independent of the frequency. The integrals over the frequencies can be easily calculated by using

$$
\frac{1}{\omega-\omega_{0} \pm \mathrm{i} \varepsilon}=\mathrm{PP} \frac{1}{\omega-\omega_{0}} \mp \mathrm{i} \pi \delta\left(\omega-\omega_{0}\right)
$$

and gives gain the classical expression

$$
\Delta E=\frac{1}{\left(4 \pi \epsilon_{0}\right)^{2}} \sum_{\text {ij }} \sum_{m, n} \frac{\left\langle 0_{A} 0_{B}\left|d_{A} T_{A B} d_{B}\right| m_{A} n_{B}\right\rangle\left\langle m_{A} n_{B}\left|d_{A} T_{A B} d_{B}\right| 0_{A} 0_{B}\right\rangle}{2 E_{0}-E_{m}-E_{n}}
$$


The scalar component $\left\{\mathrm{D}_{\mathrm{ik}} \mathrm{D}_{\mathrm{jl}}\right\}^{(0)}$ of $\mathrm{D}_{\mathrm{ik}} \mathrm{D}_{\mathrm{j} 1}$ is

$$
\left\{D_{i k} D_{j 1}\right\}^{(0)}=\delta_{i k} \delta_{j 1} \frac{2}{3}\left(3-6 x+5 x^{2}-2 x^{3}+x^{4}\right) \exp (2 x)
$$

with $x=i \omega R / c$.

In the quasi static limit, the scalar contribution of $\mathrm{D}_{\mathrm{ip}} \mathrm{D}_{\mathrm{jq}}$ is

$$
\mathrm{D}_{\mathrm{ip}} \mathrm{D}_{\mathrm{jq}} \rightarrow \frac{4}{5} \delta_{\mathrm{ip}} \delta_{\mathrm{jq}}
$$

so that

$$
\Delta E=\frac{-1}{2 \pi} \operatorname{Im}\left(\frac{4}{5 R^{6}} \int_{0}^{+\infty} \mathrm{d} \omega \sum_{\mathrm{ij}} \alpha_{\mathrm{ij}}(\omega)_{\mathrm{B}} \alpha_{\mathrm{ij}}(\omega)_{\mathrm{A}}\right)
$$

which is equivalent to

$$
\Delta E=\frac{4}{5 R^{6}} \frac{1}{\left(4 \pi \epsilon_{0}\right)^{2}} \sum_{i j} \sum_{m, n} \frac{\left\langle 0\left|d_{i} P_{m} d_{j}\right| 0\right\rangle\left\langle 0\left|d_{i} P_{m} d_{j}\right| 0\right\rangle}{2 E_{0}-E_{m}-E_{n}}
$$

In the long distance limit, equ.(A1.4) is approximated by

$$
\Delta \mathrm{E} \simeq \frac{-1}{2 \pi} \operatorname{Im}\left(\sum_{\mathrm{ijpq}} \alpha_{\mathrm{ij}}(0)_{\mathrm{B}} \alpha_{\mathrm{pq}}(0)_{\mathrm{A}} \mathrm{R}^{-6} \int_{0}^{+\infty} \hbar d \omega \mathrm{D}_{\mathrm{ip}}(\mathrm{i} \omega \mathrm{R} / \mathrm{c}) \mathrm{D}_{\mathrm{jq}}(\mathrm{i} \omega \mathrm{R} / \mathrm{c})\right)
$$

The scalar contribution of $\mathrm{D}_{\mathrm{ip}} \mathrm{D}_{\mathrm{jq}}$ can be checked to be equal to

$$
\frac{2}{3}\left(3-6 x+5 x^{2}-2 x^{3}+x^{4}\right) \exp (2 x)
$$

so that

$$
\Delta \mathrm{E} \simeq \frac{-1}{2 \pi} \operatorname{Im}\left(\sum_{\mathrm{ijpq}} \alpha_{\mathrm{ij}}(0)_{\mathrm{B}} \alpha_{\mathrm{ij}}(0)_{\mathrm{A}} \mathrm{R}^{-6} \int_{0}^{+\infty} \hbar d \omega \frac{2}{3}\left(3-6 \mathrm{x}+5 \mathrm{x}^{2}-2 \mathrm{x}^{3}+\mathrm{x}^{4}\right) \exp (2 \mathrm{x})\right)
$$

The integral can be exactly calculated by putting $\xi=-i \omega / \mathrm{R} / \mathrm{c}$ and an integration over the contour of fig.(3) and it finally leads to 


$$
\Delta \mathrm{E}=\frac{23 \hbar \mathrm{c}}{12 \pi}\left(\sum_{\mathrm{ijpq}} \alpha_{\mathrm{ij}}(0)_{\mathrm{B}} \alpha_{\mathrm{ij}}(0)_{\mathrm{A}} \mathrm{R}^{-7}\right)
$$




\section{Annexe 2}

The transition amplitude for the 1st diagram of fig.(3) is

$$
\begin{aligned}
& \mathrm{T}_{\mathrm{i} \rightarrow \mathrm{f}}=\lim _{\varepsilon, \varepsilon^{\prime}, \varepsilon^{\prime \prime}, \varepsilon_{A}, \varepsilon_{A}^{\prime}, \varepsilon_{B}, \varepsilon_{B}^{\prime} \rightarrow 0} \int_{-\infty}^{+\infty} d \mathrm{t}_{6} \int_{-\infty}^{+\infty} d \mathrm{t}_{5} \int_{-\infty}^{+\infty} d \mathrm{t}_{4} \int_{-\infty}^{+\infty} d \mathrm{t}_{3} \int_{-\infty}^{+\infty} d \mathrm{t}_{2} \int_{-\infty}^{+\infty} d \mathrm{t}_{1} \\
& (\mathrm{i} / 2 \pi)^{7} \int d \mathrm{E}^{\prime}{ }_{\mathrm{A}} \int d \mathrm{E}_{\mathrm{A}} \int d \mathrm{E}^{\prime}{ }_{\mathrm{B}} \int d \mathrm{E}_{\mathrm{B}} \int d\left(\omega^{\prime \prime 2}\right) \int d\left(\omega^{\prime 2}\right) \int d\left(\omega^{2}\right) \\
& \exp -i\left(t_{f}-t_{3}\right) E_{0} \hbar^{-1} \cdot \exp -i\left(t_{3}-t_{2}\right) E_{A}^{\prime} \hbar^{-1} \cdot \exp -i\left(t_{2}-t_{1}\right) E_{A} \hbar^{-1} \cdot \exp -i\left(t_{1}-t_{i}\right) E_{0} \hbar^{-1} \\
& \exp -\mathrm{i}\left(\mathrm{t}_{\mathrm{f}}-\mathrm{t}_{6}\right) \mathrm{E}_{0} \hbar^{-1} \cdot \exp -\mathrm{i}\left(\mathrm{t}_{6}-\mathrm{t}_{5}\right) \mathrm{E}_{\mathrm{B}}^{\prime} \hbar^{-1} \cdot \exp -\mathrm{i}\left(\mathrm{t}_{5}-\mathrm{t}_{4}\right) \mathrm{E}_{\mathrm{B}} \hbar^{-1} \cdot \exp -\mathrm{i}\left(\mathrm{t}_{4}-\mathrm{t}_{\mathrm{i}}\right) \mathrm{E}_{0} \hbar^{-1} \\
& \iiint \frac{\mathrm{V} d^{3} \mathbf{k} "}{(2 \pi)^{3}} \frac{1}{\omega^{\prime \prime 2}-c^{2} k^{\prime 2}+\mathrm{i} \varepsilon^{\prime \prime}} \frac{\hbar \omega^{\prime \prime}}{2 \epsilon_{0} \mathrm{~V}} \operatorname{expik} " . \mathbf{R}_{\mathrm{AB}} \sum_{\mathbf{e}^{\prime \prime \perp \mathbf{k}}} \mathrm{e}{ }_{\mathrm{u}} \mathrm{e}{ }_{\mathrm{V}}
\end{aligned}
$$

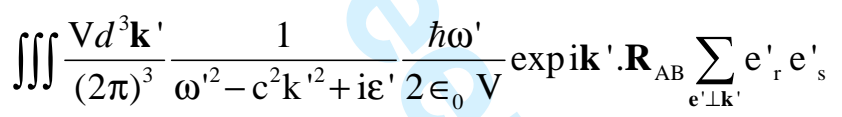

$$
\begin{aligned}
& \iiint \frac{\mathrm{V} d^{3} \mathbf{k}}{(2 \pi)^{3}} \frac{1}{\omega^{2}-\mathrm{c}^{2} \mathrm{k}^{2}+\mathrm{i} \varepsilon} \frac{\hbar \omega}{2 \epsilon_{0} \mathrm{~V}} \exp -\mathrm{ik} \cdot \mathbf{R}_{\mathrm{AB}} \sum_{\mathbf{e} \perp \mathbf{k}} \mathrm{e}_{\mathrm{p}} \mathrm{e}_{\mathrm{q}} \\
& \sum_{n, n^{\prime}} \frac{\left\langle 0\left|d_{v}(B)\right| n^{\prime}\right\rangle\left\langle n^{\prime}\left|d_{s}(B)\right| n\right\rangle\left\langle n\left|d_{q}(B)\right| 0\right\rangle}{\left(E_{B}^{\prime}-\hbar \omega^{\prime \prime}+i \varepsilon_{B}^{\prime}\right)\left(E_{0}-E_{B}-\hbar \omega+i \varepsilon_{B}\right)} \\
& \sum_{m, m^{\prime}} \frac{\left\langle 0\left|d_{u}(A)\right| n^{\prime}\right\rangle\left\langle n^{\prime}\left|d_{r}(A)\right| n\right\rangle\left\langle n\left|d_{p}(A)\right| 0\right\rangle}{\left(E_{0}-E_{A}^{\prime}+\hbar \omega^{\prime \prime}+i \varepsilon^{\prime}{ }_{A}\right)\left(E_{0}-E_{A}+\hbar \omega+i \varepsilon_{A}\right)}
\end{aligned}
$$

The other diagrams can be expressed in a similar way. We get

$$
\begin{aligned}
& \mathrm{T}_{\mathrm{i} \rightarrow \mathrm{f}}=\lim _{\varepsilon, \varepsilon^{\prime}, \varepsilon^{\prime \prime} \rightarrow 0}(-\mathrm{i})\left(\hbar / 4 \pi^{2}\right)\left(\mathrm{t}_{\mathrm{f}}-\mathrm{t}_{\mathrm{i}}\right) \exp \left[-\mathrm{iE}_{0}\left(\mathrm{t}_{\mathrm{f}}-\mathrm{t}_{\mathrm{i}}\right) \hbar^{-1}\right] \\
& \int_{0}^{\infty} \omega^{\prime \prime 2} d \omega^{\prime \prime} \int_{0}^{\infty} \omega^{\prime 2} d \omega^{\prime} \int_{0}^{\infty} \omega^{2} d \omega \quad \delta\left(\omega^{\prime \prime}-\omega^{\prime}-\omega\right) \\
& \epsilon_{0}^{-3} \sum_{n, n^{\prime}} \frac{\left\langle 0\left|d_{v}(B)\right| n^{\prime}\right\rangle\left\langle n^{\prime}\left|d_{s}(B)\right| n\right\rangle\left\langle n\left|d_{q}(B)\right| 0\right\rangle}{\left(E_{0}-E_{n^{\prime}}-\hbar \omega^{\prime \prime}+i \varepsilon_{B}^{\prime}\right)\left(E_{0}-E_{n}-\hbar \omega+i \varepsilon_{B}\right)} \sum_{m, m^{\prime}} \frac{\left\langle 0\left|d_{u}(A)\right| n^{\prime}\right\rangle\left\langle n^{\prime}\left|d_{r}(A)\right| n\right\rangle\left\langle n\left|d_{p}(A)\right| 0\right\rangle}{\left(E_{0}-E_{n^{\prime}}+\hbar \omega^{\prime \prime}+i \varepsilon_{A}^{\prime}\right)\left(E_{0}-E_{n}+\hbar \omega+i \varepsilon_{A}\right)} \\
& \iiint \frac{d^{3} \mathbf{k}^{\prime \prime}}{(2 \pi)^{3}} \frac{1}{\omega^{\prime \prime 2}-\mathrm{c}^{2} \mathrm{k}^{\prime \prime}+\mathrm{i} \varepsilon^{\prime \prime}} \iiint \frac{d^{3} \mathbf{k}^{\prime}}{(2 \pi)^{3}} \frac{1}{\omega^{\prime 2}-\mathrm{c}^{2} \mathrm{k}^{\prime 2}+\mathrm{i} \varepsilon^{1}} \iiint \frac{d^{3} \mathbf{k}}{(2 \pi)^{3}} \frac{1}{\omega^{2}-\mathrm{c}^{2} \mathrm{k}^{2}+\mathrm{i} \varepsilon} \\
& \operatorname{expi}\left(\mathbf{k}+\mathbf{k}^{\prime}-\mathbf{k} "\right) \cdot \mathbf{R}_{\mathrm{AB}} \sum_{\mathbf{e}^{\prime \prime} \perp \mathbf{k}^{\prime \prime}} \mathrm{e}^{\prime \prime}{ }_{\mathrm{u}} \mathrm{e}^{\prime}{ }_{\mathrm{v}} \sum_{\mathbf{e}^{\prime} \perp \mathbf{k}^{\prime}} \mathrm{e}_{\mathrm{r}}^{\prime} \mathrm{e}^{\prime}{ }_{\mathrm{e} \perp \mathbf{k}} \mathrm{e}_{\mathrm{p}} \mathrm{e}_{\mathrm{q}}
\end{aligned}
$$


The total transition amplitude is $T_{i \rightarrow f}=T_{i \rightarrow f}^{0}+T_{i \rightarrow f}$ where $\mathrm{T}_{\mathrm{i} \rightarrow \mathrm{f}}^{0}=\exp \left[-\mathrm{iE}_{0}\left(\mathrm{t}_{\mathrm{f}}-\mathrm{t}_{\mathrm{i}}\right) \hbar^{-1}\right]$ accounts for the trivial un-perturbated case.

It is easy to check that $\sum_{\mathbf{e} \perp \mathbf{k}} \mathrm{e}_{\mathrm{j}} \mathrm{e}_{1}=\delta_{\mathrm{jl}}-\frac{\mathrm{k}_{\mathrm{j}} \mathrm{k}_{1}}{\mathrm{k}^{2}}$.

Then we have to calculate the two integrals

$\lim _{\varepsilon \rightarrow 0} \iiint \frac{d^{3} \mathbf{k}}{(2 \pi)^{3}} \frac{1}{\omega^{2}-c^{2} k^{2}+i \varepsilon} \exp -i \mathbf{k} \cdot \mathbf{R}_{A B}$

and

$\lim _{\varepsilon \rightarrow 0} \iiint \frac{\mathrm{d}^{3} \mathbf{k}}{(2 \pi)^{3}} \frac{\mathrm{k}_{\mathrm{j}} \mathrm{k}_{1}}{\mathrm{k}^{2}} \frac{1}{\omega^{2}-\mathrm{c}^{2} \mathrm{k}^{2}+\mathrm{i} \varepsilon} \exp -\mathrm{ik} \cdot \mathbf{R}_{\mathrm{AB}}$

The second integral is

$\lim _{\varepsilon \rightarrow 0}-\frac{c^{2}}{\omega^{2}} \nabla_{j} \nabla_{1} \iiint \frac{d^{3} \mathbf{k}}{(2 \pi)^{3}} \frac{1}{\omega^{2}-c^{2} k^{2}+i \varepsilon} \exp -i k \cdot R_{A B}$

and the first integral is

$\lim _{\varepsilon \rightarrow 0} \frac{4 \pi}{(2 \pi)^{3}} \int_{-\infty}^{+\infty} \frac{k^{2} d k}{\omega^{2}-c^{2} k^{2}+i \varepsilon} \frac{\operatorname{expikR}}{2 i k R}=-\frac{\operatorname{expi\omega R} / c}{4 \pi c^{2} R}$

We finally obtain

$$
\begin{aligned}
& \lim _{\varepsilon \rightarrow 0} \iiint \frac{d^{3} \mathbf{k}}{(2 \pi)^{3}} \frac{1}{\omega^{2}-\mathrm{c}^{2} \mathrm{k}^{2}+\mathrm{i \varepsilon}} \exp -\mathrm{ik} \cdot \mathbf{R}_{\mathrm{AB}} \sum_{\mathbf{e} \perp \mathbf{k}} \mathrm{e}_{\mathrm{j}} \mathrm{e}_{1}=-\left(\delta_{\mathrm{j} 1}+\frac{\mathrm{c}^{2}}{\omega^{2}} \nabla_{\mathrm{j}} \nabla_{1}\right)\left(\frac{\operatorname{expi\omega R/c}}{4 \pi \mathrm{c}^{2} \mathrm{R}}\right) \\
& ==\delta_{\mathrm{j} 1}\left(1-\left(\frac{\mathrm{i} \omega \mathrm{R}}{\mathrm{c}}\right)+\left(\frac{\mathrm{i} \omega \mathrm{R}}{\mathrm{c}}\right)^{2}\right)\left(\frac{\operatorname{expi\omega R/c}}{4 \pi \omega^{2} \mathrm{R}^{3}}\right)-\frac{\mathrm{R}_{\mathrm{j}} \mathrm{R}_{1}}{\mathrm{R}^{2}}\left(3-3\left(\frac{\mathrm{i} \omega \mathrm{R}}{\mathrm{c}}\right)+\left(\frac{\mathrm{i} \omega \mathrm{R}}{\mathrm{c}}\right)^{2}\right)\left(\frac{\operatorname{expi\omega R/c}}{4 \pi \omega^{2} \mathrm{R}^{3}}\right)
\end{aligned}
$$

Let us put 


$$
\begin{aligned}
D_{j 1}(x) & =\delta_{j 1}\left(1-x+x^{2}\right) \exp (x)-\frac{R_{j} R_{1}}{R^{2}}\left(3-3 x+x^{2}\right) \exp (x) \\
& =\delta_{j 1} \frac{2}{3} x^{2} \exp (x)-\left(\frac{3 R_{j} R_{1}-R^{2} \delta_{j 1}}{2 R^{2}}\right) \frac{2}{3}\left(3-3 x+x^{2}\right) \exp (x)
\end{aligned}
$$

We obtain

$$
\begin{aligned}
\mathrm{T}_{\mathrm{i} \rightarrow \mathrm{f}}= & \lim _{\varepsilon, \varepsilon^{\prime}, \varepsilon^{\prime \prime} \rightarrow 0}(-\mathrm{i})\left(\hbar / 4 \pi^{2}\right)\left(\mathrm{t}_{\mathrm{f}}-\mathrm{t}_{\mathrm{i}}\right) \exp \left[-\mathrm{iE}_{0}\left(\mathrm{t}_{\mathrm{f}}-\mathrm{t}_{\mathrm{i}}\right) \hbar^{-1}\right] \\
& \mathrm{R}^{-9} \int_{0}^{\infty} \omega^{\prime \prime 2} d \omega^{\prime \prime} \int_{0}^{\infty} \omega^{\prime 2} d \omega^{\prime} \int_{0}^{\infty} \omega^{2} d \omega \delta\left(\omega^{\prime \prime}-\omega^{\prime}-\omega\right) \mathrm{D}_{\mathrm{vu}}(\mathrm{x}) \mathrm{D}_{\mathrm{sr}}(\mathrm{x}) \mathrm{D}_{\mathrm{qp}}(\mathrm{x}) \\
& \epsilon_{0}^{-3} \sum_{\mathrm{n}, \mathrm{n}^{\prime}} \frac{\left\langle 0\left|\mathrm{~d}_{\mathrm{v}}(\mathrm{B})\right| \mathrm{n}^{\prime}\right\rangle\left\langle\mathrm{n}^{\prime}\left|\mathrm{d}_{\mathrm{s}}(\mathrm{B})\right| \mathrm{n}\right\rangle\left\langle\mathrm{n}\left|\mathrm{d}_{\mathrm{q}}(\mathrm{B})\right| 0\right\rangle}{\left.\mathrm{E}_{\mathrm{n}^{\prime}}-\hbar \omega^{\prime \prime}+\mathrm{i} \varepsilon_{\mathrm{B}}^{\prime}\right)\left(\mathrm{E}_{0}-\mathrm{E}_{\mathrm{n}}-\hbar \omega+\mathrm{i} \varepsilon_{\mathrm{B}}\right)} \sum_{\mathrm{m}, \mathrm{m}^{\prime}} \frac{\left\langle 0\left|\mathrm{~d}_{\mathrm{u}}(\mathrm{A})\right| \mathrm{n}^{\prime}\right\rangle\left\langle\mathrm{n}^{\prime}\left|\mathrm{d}_{\mathrm{r}}(\mathrm{A})\right| \mathrm{n}\right\rangle\left\langle\mathrm{n}\left|\mathrm{d}_{\mathrm{p}}(\mathrm{A})\right| 0\right\rangle}{\left(\omega^{\prime}+\mathrm{i}^{\prime}{ }_{\mathrm{A}}\right)\left(\mathrm{E}_{0}-\mathrm{E}_{\mathrm{n}}+\hbar \omega+\mathrm{i} \varepsilon_{\mathrm{A}}\right)}
\end{aligned}
$$




\section{Annexe 3}

The 4 terms described by the diagrams of fig. 3 are resonant at the frequency $\omega$ and involve the integrals

$$
\begin{aligned}
\int_{0}^{\infty} \frac{d \omega}{\left(E_{0}-\right.} & \left.E_{n}+\hbar \omega+i \varepsilon\right)\left(E_{0}-E_{n^{\prime}}-\hbar \omega-i \varepsilon\right) \\
\quad= & \frac{1}{\left(E_{0}-E_{n^{\prime}}\right)+\left(E_{0}-E_{n}\right)} \int_{0}^{\infty} d \omega\left[\frac{1}{\left(E_{0}-E_{n}+\hbar \omega+i \varepsilon\right)}+\frac{1}{\left(E_{0}-E_{n^{\prime}}-\hbar \omega-i \varepsilon\right)}\right] \\
= & \frac{1}{\left(2 E_{0}-E_{n}-E_{n^{\prime}}\right)} \log \left(\frac{E_{0}-E_{n^{\prime}}}{E_{0}-E_{n}}\right)-\frac{i \pi}{\left(2 E_{0}-E_{n}-E_{n^{\prime}}\right)}
\end{aligned}
$$

But

$$
\sum_{n, n^{\prime}} \frac{1}{\left(2 E_{0}-E_{n}-E_{n^{\prime}}\right)} \log \left(\frac{E_{0}-E_{n^{\prime}}}{E_{0}-E_{n}}\right)=0
$$

so that

$$
\sum_{n, n^{\prime}} \int_{0}^{\infty} \frac{d \omega}{\left(E_{0}-E_{n}+\hbar \omega+i \varepsilon\right)\left(E_{0}-E_{n^{\prime}}-\hbar \omega-i \varepsilon\right)}=\frac{-i \pi}{\left(2 E_{0}-E_{n}-E_{n^{\prime}}\right)}
$$

Hence

$$
\begin{aligned}
\sum_{m, m^{\prime}, n, n^{\prime}} \int_{0}^{\infty} & \frac{d \omega}{\left(E_{0}-E_{n}+\hbar \omega+i \varepsilon\right)\left(E_{0}-E_{n^{\prime}}-\hbar \omega-i \varepsilon\right)} \int_{0}^{\infty} \frac{d \omega^{\prime}}{\left(E_{0}-E_{m}+\hbar \omega^{\prime}+i \varepsilon^{\prime}\right)\left(E_{0}-E_{m^{\prime}}-\hbar \omega^{\prime}-i \varepsilon^{\prime}\right)} \\
& =\sum_{m, m^{\prime}, n, n^{\prime}} \frac{-\pi^{2}}{\left(2 E_{0}-E_{n}-E_{n^{\prime}}\right)\left(2 E_{0}-E_{m}-E_{m^{\prime}}\right)}
\end{aligned}
$$




\section{Figure caption:}

Fig.1: Diagrams expressing the first order non linear optical phenomena (frequency sum and difference).

Fig.2: The 3 types of topologically different diagrams expressing the interaction of the 2 molecules A and B via the exchange of 3 virtual photons.

Fig.3: The 4 diagrams actually contributing to the interaction energy.

Fig.4: Relative configurations of the molecular planes: case of intersecting molecular planes.

Fig.5: Relative configurations of the molecular planes: case of parallel molecular planes. 
Fig.1

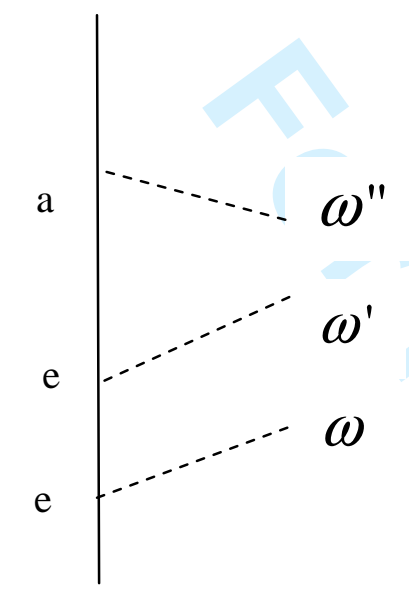

A

Fig. 1a

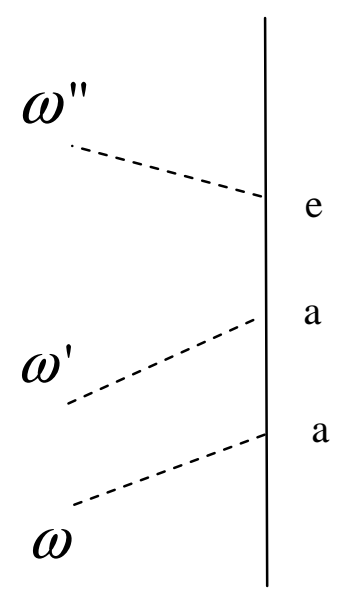

A Fig. 1b 
Fig. 2

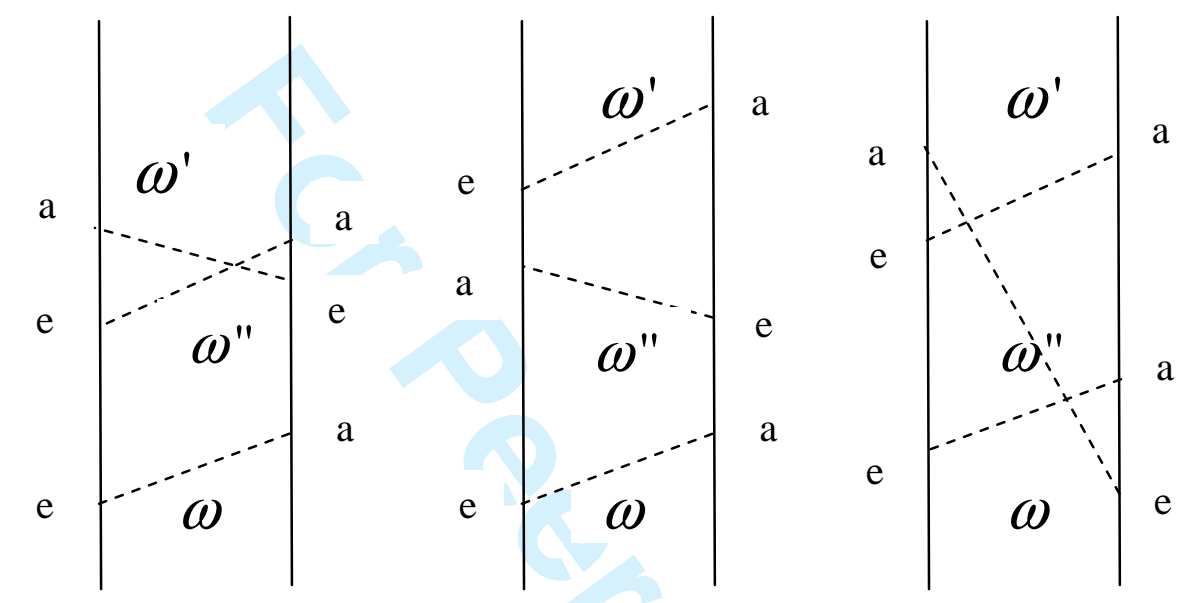


Fig.3

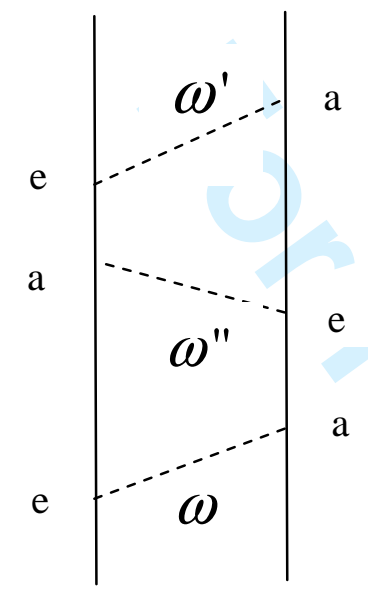

A $\quad$ B

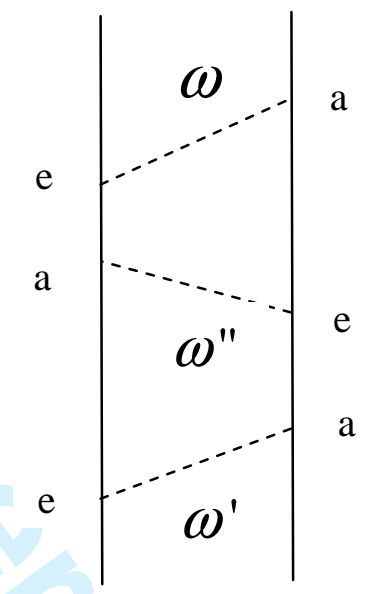

A $\quad$ B

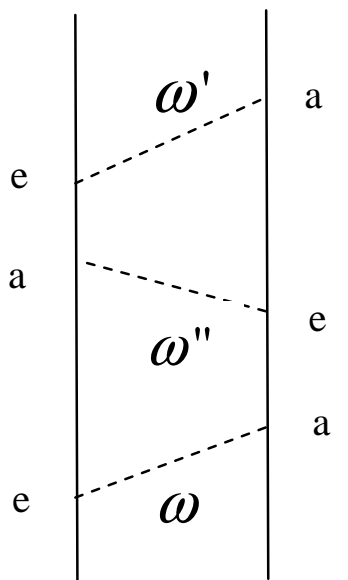

B

A

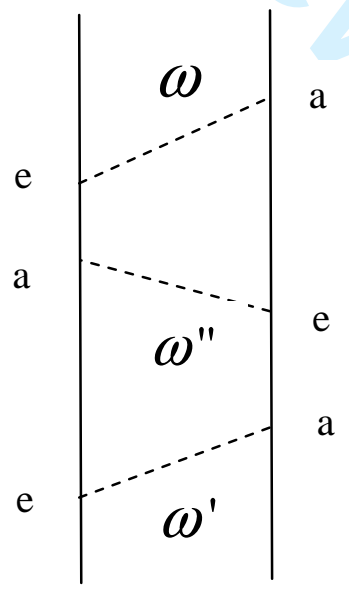

B A 
Fig. 4

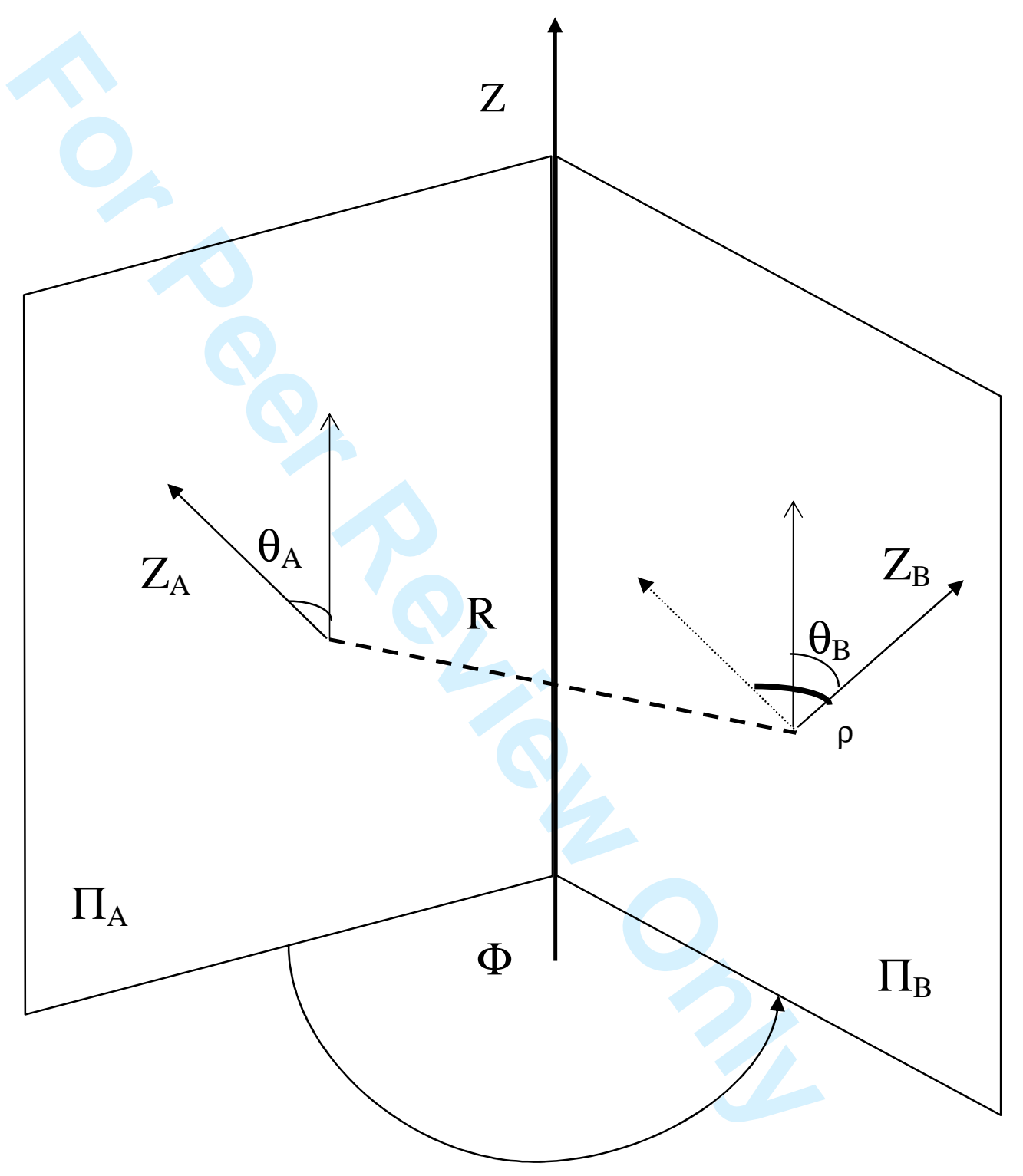


Fig. 5

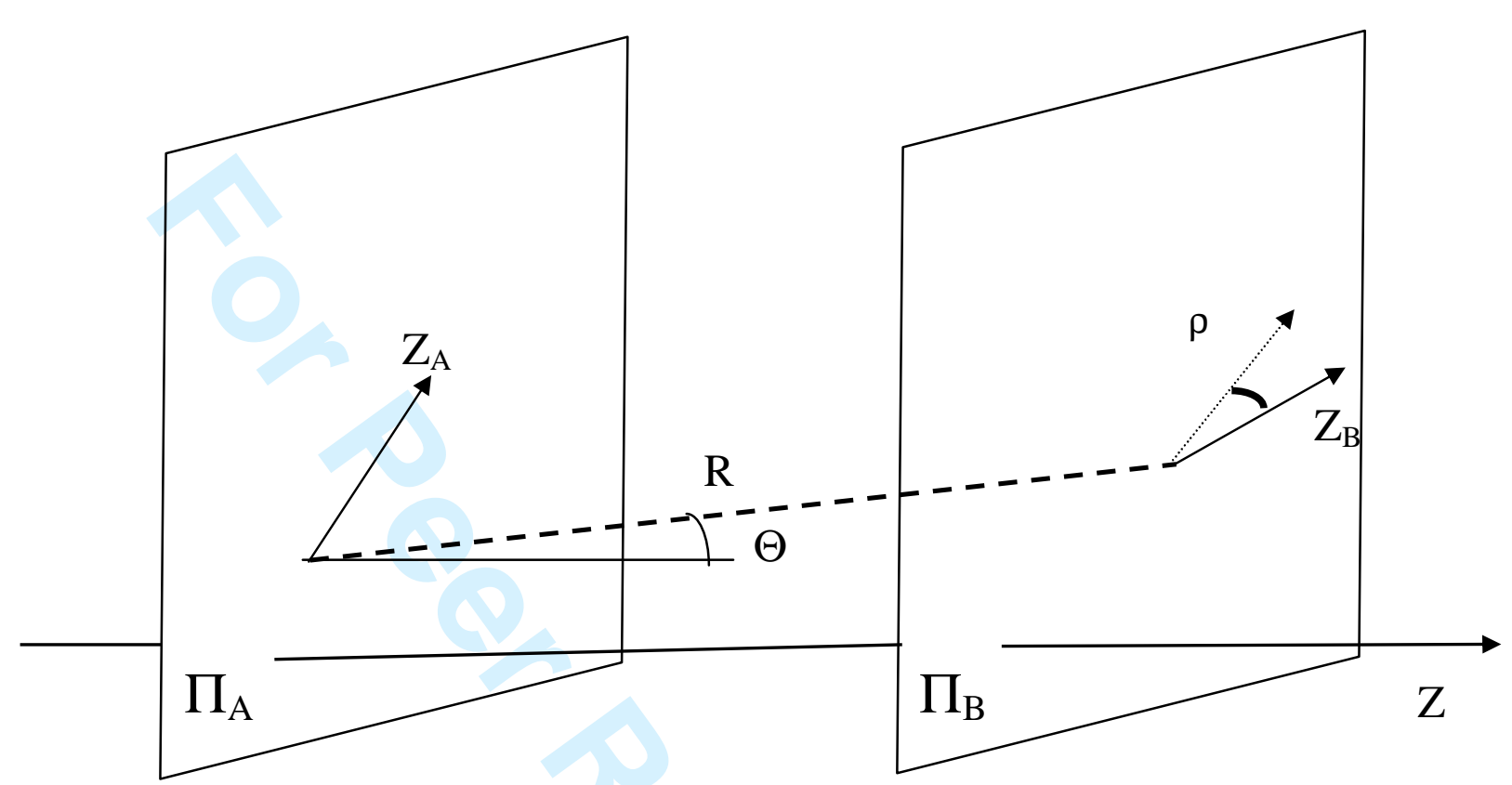


References:

[1] L. Landau, E. Lifshitz, Electrodynamics of continuous media (MIR, Moscow, 1969)

[2] N. Bloembergen, Nonlinear optics (Benjamin, New-York, 1965)

[3] R. Bonneville, Molecular Physics, 106, 2627 (2008) and references therein

[4] P.E. Schipper, Chem. Phys. 26, 29 (1977)

[5] R.A. Harris, Mol. Phys. 105, 2433 (2007)

[6] R. Bonneville, Mol.Cryst.Liq.Cryst., Sci.Technol.-Sec.B : Nonlinear Optics, 2, 159 (1992)

[7] A.R. Edmonds, Angular momentum in quantum mechanics (Princeton University Press, Princeton, 1960) 


\title{
Comparison of the $3^{\text {rd }}$ order dispersion terms of interaction between chiral molecules
}

\author{
Richard BONNEVILLE \\ CNES, 2 place Maurice Quentin, 75001 Paris, France \\ 33.80.-b, 33.55.Ad, 33.15.-e, 33.00.00, 34.20.Gj, 61.30.-v, 61.30.Gd
}

\begin{abstract}
Through a quantum electrodynamic approach, we investigate the coupling between chiral molecules deriving from the exchange of 3 virtual photons of the electric dipole type. The resulting interaction potential, which involves the first nonlinear polarizability of the molecules, is compared with a previously derived interaction potential involving the optical activity of the molecules; the latter is accounted for by the exchange of 2 virtual photons, one being electric dipolar and the other being electric quadrupolar or magnetic dipolar. The symmetry properties of the new term is considered and its quasi static limit is derived. It appears that the two interaction terms, which both are third-order dispersion processes, might have the same order of magnitude; thus none can a priori be neglected and both might equally contribute to the possibility of an helical ordering.
\end{abstract}




\section{Introduction}

When they are coupled with the electromagnetic field, chiral molecules exhibit two noticeable characteristics: (i) they are optically active, (ii) they have a non-zero first non linear polarizability. The rank-3 true tensor $\eta_{\text {ipj }}(\omega)$ which accounts for optical activity is anti-symmetric in the exchange of its extreme indices $\mathrm{i}$ and $\mathrm{j}$ from a general property of the kinetic coefficients and thus it is equivalent to a rank-2 pseudo-tensor [1]. The first non linear polarizability responsible for the parametric optical phenomena at molecular level [2] is expressed by rank-3 true tensor $\beta_{\mathrm{ijk}}\left(\omega_{1}, \omega_{2}, \omega_{3}\right)$ with $\omega_{1}+\omega_{2}+\omega_{3}=0$.

In a recent paper [3] we had reviewed the symmetries of the electromagnetic interactions between chiral molecules. Molecular interactions include longitudinal static couplings between permanent dipoles and transverse dynamic couplings; in the quantum electrodynamics formulation of the field - matter interactions the latter are viewed as resulting from an exchange of virtual photons between the molecules.

The lowest order term of those dynamic interactions involves four coupling vortices and the exchange of two photons. If those elementary couplings are of electric dipole type, we obtain the well-known Van der Waals interaction which can be expressed as a function of the linear polarizability $\alpha$ of the two molecules and which varies as $\mathrm{R}^{-6}$ in the short range limit. If we go beyond the electric dipole approximation and consider magnetic dipole or electric quadrupole type couplings, additional interaction terms can be evidenced between chiral molecules, with a $\mathrm{R}^{-8}$ dependence in the short range limit. Those chiral terms can be expressed as a function of the optical activity $\eta$ of the two molecules. 
The association of the anisotropic part of those chiral terms with the anisotropic part of the Van der Waals interaction can account for the helicoidal order of the chiral nematics (cholesteric phases). The anisotropic Van der Waals interaction can be expressed as a function of the scalar product $\alpha^{(2)}(\mathrm{A}) \cdot \alpha^{(2)}(\mathrm{B})$ of the quadrupolar components of the linear polarizabilities, and the anisotropic chiral interaction can be expressed as a function of the scalar product $\eta^{(2)}(\mathrm{A}) \cdot \eta^{(2)}(\mathrm{B})$ of the quadrupolar components of the optical activities. Both interactions have the same rotational symmetry properties, but the Van der Waals term is always attractive and tends to align the molecules parallel or antiparallel to one of the main axes of $\alpha^{(2)}$, conversely the chiral term is always repulsive and tends to align the molecules perpendicular to one of the main axes of $\eta^{(2)}$ (N.B.: the main axes of the molecular tensors $\alpha^{(2)}$ and $\eta^{(2)}$ are a priori different). The 2 terms thus tend to align the molecules along two different directions; it is well known that a system undergoing forces with different symmetries and ranges may minimise its free energy by adopting an helical ordering. The $\mathrm{Z}$ axis of $\alpha^{(2)}$ and the $\mathrm{Z}$ axis of $\eta^{(2)}$ define a molecular plane $\Pi$. The interaction energy between 2 molecules $A$ and $B$ is minimal if either the molecular planes $\Pi_{\mathrm{A}}$ and $\Pi_{\mathrm{B}}$ are identical, i.e. there is a nematic-type order in that plane, or if $\Pi_{\mathrm{A}}$ and $\Pi_{\mathrm{B}}$ are parallel, the nematic axes of the 2 planes being shifted by a certain angle, which generates a cholesteric-type twist.

\section{Nonlinear coupling between chiral molecules}


In the quasi-static limit (Appendix 1) the dynamical expression of the Van der Waals forces gives again the classical expression derived from a static dipole-dipole interaction at the $2^{\text {nd }}$ order of perturbation:

$$
\mathrm{V}_{\mathrm{AB}}=\sum_{\mathrm{m}, \mathrm{n}} \frac{\left\langle 0_{\mathrm{A}} 0_{\mathrm{B}}\left|\mathrm{d}_{\mathrm{A}} \mathrm{T}_{\mathrm{AB}} \mathrm{d}_{\mathrm{B}}\right| \mathrm{m}_{\mathrm{A}} \mathrm{n}_{\mathrm{B}}\right\rangle\left\langle\mathrm{m}_{\mathrm{A}} \mathrm{n}_{\mathrm{B}}\left|\mathrm{d}_{\mathrm{A}} \mathrm{T}_{\mathrm{AB}} \mathrm{d}_{\mathrm{B}}\right| 0_{\mathrm{A}} 0_{\mathrm{B}}\right\rangle}{2 \mathrm{E}_{0}-\mathrm{E}_{\mathrm{m}}-\mathrm{E}_{\mathrm{n}}}
$$

where $T_{A B}$ is the static dipole-dipole coupling tensor, leading to the R-dependence in $\mathrm{R}^{-6}$. Now it has been pointed out by Schipper [4] that a $3^{\text {rd }}$ order perturbation term of the static dipole-dipole interaction would give a non-zero contribution for chiral molecules, namely:

$$
\mathrm{V}_{\mathrm{AB}}=\sum_{\mathrm{m}, \mathrm{n}, \mathrm{m}^{\prime}, \mathrm{n}^{\prime}} \frac{\left\langle 0_{\mathrm{A}} 0_{\mathrm{B}}\left|\mathrm{d}_{\mathrm{A}} \mathrm{T}_{\mathrm{AB}} \mathrm{d}_{\mathrm{B}}\right| \mathrm{m}^{\prime}{ }_{\mathrm{A}} \mathrm{n}^{\prime}{ }_{\mathrm{B}}\right\rangle\left\langle\mathrm{m}^{\prime}{ }_{\mathrm{A}} \mathrm{n}^{\prime}{ }_{\mathrm{B}}\left|\mathrm{d}_{\mathrm{A}} \mathrm{T}_{\mathrm{AB}} \mathrm{d}_{\mathrm{B}}\right| \mathrm{m}_{\mathrm{A}} \mathrm{n}_{\mathrm{B}}\right\rangle\left\langle\mathrm{m}_{\mathrm{A}} \mathrm{n}_{\mathrm{B}}\left|\mathrm{d}_{\mathrm{A}} \mathrm{T}_{\mathrm{AB}} \mathrm{d}_{\mathrm{B}}\right| 0_{\mathrm{A}} 0_{\mathrm{B}}\right\rangle}{\left(2 \mathrm{E}_{0}-\mathrm{E}_{\mathrm{m}^{\prime}}-\mathrm{E}_{\mathrm{n}^{\prime}}\right)\left(2 \mathrm{E}_{0}-\mathrm{E}_{\mathrm{m}}-\mathrm{E}_{\mathrm{n}}\right)}
$$

with a R-dependence in $\mathrm{R}^{-9}$. Then Harris [5] have shown that this $\mathrm{R}^{-9}$ interaction could be expressed as a double integral over frequencies of a product of 2 complex sum frequency generation responses.

Actually, the expression equ.(2) above is the static limit of a dynamic process in which the calculation of the intermolecular energy expresses the exchange of 3 virtual photons between the two molecules with the 6 coupling vortices being of electric dipole type. More explicitly, 2 types of field-matter couplings can be called for each of the two molecules:

(i) either the absorption of two photons of respective frequencies $\omega$ and $\omega^{\prime}$ and the emission of one photon of frequency $\omega^{\prime \prime}=\omega+\omega^{\prime}$, that is accounted for by 6 terms obtained by permutation of the 3 vortices of fig.(1a), 
(ii) or the emission of two photons of respective frequencies $\omega$ and $\omega^{\prime}$ and the absorption of one photon of frequency $\omega^{\prime \prime}=\omega^{\prime}+\omega$, that is accounted for by 6 terms obtained by permutation of the 3 vortices of fig.(1b).

The nonlinear interaction between molecule A and molecule B is thus accounted for either by process (i) for molecule A associated to process (ii) for molecule B, i.e. 36 terms, or in a symmetric way by process (ii) for molecule A associated to process (i) for molecule B, i.e. 36 terms. Finally, there are 72 terms in total describing the whole process, each belonging to one of the 3 topological types of fig. 2 .

By following the same approach as in ref.[3], the 3-photons transition amplitude after integration over the 6 interaction times between an initial instant $t_{i} \rightarrow-\infty$ when the field - matter coupling is switched on and a final instant $t_{f} \rightarrow+\infty$ where it is switched off can be expressed (Appendix 2) as

$$
\begin{aligned}
\mathrm{T}_{\mathrm{i} \rightarrow \mathrm{f}}= & \mathrm{T}_{\mathrm{i} \rightarrow \mathrm{f}}^{0}+\left( \pm \mathrm{i} \hbar / 4 \pi^{2}\right)\left(\mathrm{t}_{\mathrm{f}}-\mathrm{t}_{\mathrm{i}}\right)\left(4 \pi \in_{0}\right)^{-3} \mathrm{R}^{-9} \exp \left[-\mathrm{iE}_{0}\left(\mathrm{t}_{\mathrm{f}}-\mathrm{t}_{\mathrm{i}}\right) \hbar^{-1}\right] \int_{0}^{+\infty} d \omega \int_{0}^{+\infty} d \omega^{\prime} \\
& \sum_{\mathrm{ijkpqr}}\left(\begin{array}{l}
\beta_{\text {urp }}\left(-\omega^{\prime}-\omega, \omega^{\prime}, \omega\right)_{\mathrm{A}} \beta_{\mathrm{vsq}}\left(\omega^{\prime}+\omega,-\omega^{\prime},-\omega\right)_{\mathrm{B}} \mathrm{D}_{\mathrm{uv}}\left(\mathrm{i}\left(\omega^{\prime}+\omega\right) \mathrm{R} / \mathrm{c}\right) \mathrm{D}_{\mathrm{rs}}\left(\mathrm{i} \omega^{\prime} \mathrm{R} / \mathrm{c}\right) \mathrm{D}_{\mathrm{pq}}(\mathrm{i} \omega \mathrm{R} / \mathrm{c}) \\
+\beta_{\text {urp }}\left(\omega^{\prime}+\omega,-\omega^{\prime},-\omega\right)_{\mathrm{A}} \beta_{\mathrm{vsq}}\left(-\omega^{\prime}-\omega, \omega^{\prime}, \omega\right)_{\mathrm{B}} \mathrm{D}_{\mathrm{uv}}\left(-\mathrm{i}\left(\omega^{\prime}+\omega\right) \mathrm{R} / \mathrm{c}\right) \mathrm{D}_{\mathrm{rs}}\left(-\mathrm{i} \omega^{\prime} \mathrm{R} / \mathrm{c}\right) \mathrm{D}_{\mathrm{pq}}(-\mathrm{i} \omega \mathrm{R} / \mathrm{c})
\end{array}\right.
\end{aligned}
$$

$\mathrm{T}_{\mathrm{i} \rightarrow \mathrm{f}}^{0}=\exp \left[-\mathrm{iE}_{0}\left(\mathrm{t}_{\mathrm{f}}-\mathrm{t}_{\mathrm{i}}\right) \hbar^{-1}\right\rfloor$ accounts for the trivial un-perturbed case and $\mathrm{D}_{\mathrm{j} 1}$ is given by

$$
D_{j 1}(x)=\delta_{j 1}\left(1-x+x^{2}\right) \exp (x)-\frac{R_{j} R_{1}}{R^{2}}\left(3-3 x+x^{2}\right) \exp (x)
$$

The two nonlinear polarizability tensors $\beta_{\mathrm{ijk}}\left(-\omega^{\prime}-\omega, \omega^{\prime}, \omega\right)$ and $\beta_{\mathrm{ijk}}\left(\omega^{\prime}+\omega,-\omega^{\prime},-\omega\right)$ are naturally introduced in a symmetrical way.

Calling $\Delta \mathrm{E}$ the energy shift caused by the molecular interactions so that 


$$
\mathrm{T}_{\mathrm{i} \rightarrow \mathrm{f}}=\exp \left[-\mathrm{i}\left(\mathrm{E}_{0}+\Delta \mathrm{E}\right)\left(\mathrm{t}_{\mathrm{f}}-\mathrm{t}_{\mathrm{i}}\right) \hbar^{-1}\right] \cong\left[1-\mathrm{i} \Delta \mathrm{E}\left(\mathrm{t}_{\mathrm{f}}-\mathrm{t}_{\mathrm{i}}\right) \hbar^{-1}\right] \exp \left[-\mathrm{iE}_{0}\left(\mathrm{t}_{\mathrm{f}}-\mathrm{t}_{\mathrm{i}}\right) \hbar^{-1}\right]
$$

we get by comparing equ.(3) and equ.(5)

$$
\begin{aligned}
& \Delta \mathrm{E}=\operatorname{Re}\left(\hbar^{2} / 4 \pi^{2}\right)\left(4 \pi \in_{0}\right)^{-3} \mathrm{R}^{-9} \int_{0}^{+\infty} d \omega \int_{0}^{+\infty} d \omega^{\prime} \\
& \sum_{\mathrm{ijkpqr}}\left(\begin{array}{l}
\beta_{\text {urp }}\left(-\omega^{\prime}-\omega, \omega^{\prime}, \omega\right)_{\mathrm{A}} \beta_{\mathrm{vsq}}\left(\omega^{\prime}+\omega,-\omega^{\prime},-\omega\right)_{\mathrm{B}} \mathrm{D}_{\mathrm{uv}}\left(\mathrm{i}\left(\omega^{\prime}+\omega\right) \mathrm{R} / \mathrm{c}\right) \mathrm{D}_{\mathrm{rs}}\left(\mathrm{i} \omega^{\prime} \mathrm{R} / \mathrm{c}\right) \mathrm{D}_{\mathrm{pq}}(\mathrm{i} \omega \mathrm{R} / \mathrm{c}) \\
+\beta_{\mathrm{urp}}\left(\omega^{\prime}+\omega,-\omega^{\prime},-\omega\right)_{\mathrm{A}} \beta_{\mathrm{vsq}}\left(-\omega^{\prime}-\omega, \omega^{\prime}, \omega\right)_{\mathrm{B}} \mathrm{D}_{\mathrm{uv}}\left(-\mathrm{i}\left(\omega^{\prime}+\omega\right) \mathrm{R} / \mathrm{c}\right) \mathrm{D}_{\mathrm{rs}}\left(-\mathrm{i} \omega^{\prime} \mathrm{R} / \mathrm{c}\right) \mathrm{D}_{\mathrm{pq}}(-\mathrm{i} \omega \mathrm{R} / \mathrm{c})
\end{array}\right.
\end{aligned}
$$

The interaction energy is thus expressed as a double integral over frequencies of a product of the non linear polarizabilities of the two molecules. As far as the Rdependence is concerned, the leading term in equ.(6) decreases with distance as $\mathrm{R}^{-9}$ as expected.

$\mathrm{D}_{\mathrm{j} 1}$ can be written as

$$
\mathrm{D}_{\mathrm{j} 1}(\mathrm{x})=\delta_{\mathrm{j} 1} \frac{2}{3} \mathrm{x}^{2} \exp (\mathrm{x})-\left(\frac{3 \mathrm{R}_{\mathrm{j}} \mathrm{R}_{\mathrm{l}}-\mathrm{R}^{2} \delta_{\mathrm{j} 1}}{2 \mathrm{R}^{2}}\right) \frac{2}{3}\left(3-3 \mathrm{x}+\mathrm{x}^{2}\right) \exp (\mathrm{x})
$$

As a function of $\mathbf{R} \mathrm{D}_{\text {ip }}$ is the sum of two totally symmetric (with respect to index permutations) tensors irreducible under the rotation group, a scalar one $(\mathrm{J}=0)$ and a quadrupolar one $(J=2)$. Consequently $D_{i p} D_{j q} D_{k r}$ is the sum of terms of weight $J=0, J=2$, $\mathrm{J}=4$ and $\mathrm{J}=6$, among which, assuming an isotropic distribution of the molecular centres, we will only retain the scalar contribution $(\mathrm{J}=0)$. In terms of irreducible tensor components with respect to rotations and index permutations, $\beta_{\mathrm{ijk}}$ has the following spectrum $[3,6]$ :

$$
\{3\}\left(\beta^{(3)}+\beta^{(1)}\right),\{21\}\left(\beta^{\prime(2)}+\beta^{\prime \prime(2)}+\beta^{\prime(1)}+\beta^{\prime \prime(1)}\right),\left\{1^{3}\right\} \beta^{(0)}
$$


Assuming an isotropic distribution of the molecular centres, the intermolecular coupling has thus the following form:

$$
\begin{aligned}
& \Delta \mathrm{E} \propto \int_{0}^{+\infty} \int_{0}^{+\infty} d \omega d \omega^{\prime} \mathrm{R}^{-9} \Phi\left(\omega \mathrm{R} / \mathrm{c}, \omega^{\prime} \mathrm{R} / \mathrm{c}\right)
\end{aligned}
$$

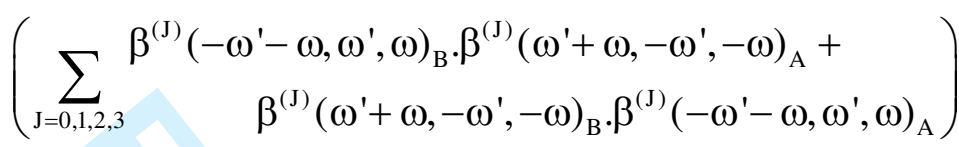

\section{Quasi static limit}

In the Miller approximation for $\beta_{\mathrm{ijk}}$ where the frequency dispersion is neglected [2], the remnant terms are the octupole-type and vector-type components $\{3\} \beta^{(3)}$ and $\{3\} \beta^{(1)}$, fully symmetric with respect to any index permutation. $\Delta \mathrm{E}$ is then essentially the sum of a vector-vector coupling and of an octupole-octupole coupling, which have to be added to the Van der Waals forces and to the chiral forces associated to optical activity.

If the retardation effects are neglected ( $\omega \mathrm{R} / \mathrm{c} \square 1$ ), then

$\mathrm{D}_{\text {ip }} \rightarrow \delta_{\text {ip }}-\frac{3 \mathrm{R}_{\mathrm{i}} \mathrm{R}_{\mathrm{p}}}{\mathrm{R}^{2}}$

i.e. $D_{\text {ip }}$ as a function of $\mathbf{R}$ is reduced to a pure quadrupole, independent of the frequency.

In the quasi static limit, the scalar (i.e. isotropic) contribution of $\mathrm{D}_{\mathrm{ip}} \mathrm{D}_{\mathrm{jq}} \mathrm{D}_{\mathrm{kr}}$ can be checked to be equal to

$\mathrm{D}_{\mathrm{ip}} \mathrm{D}_{\mathrm{jq}} \mathrm{D}_{\mathrm{kr}} \rightarrow-\frac{16}{35} \delta_{\mathrm{ip}} \delta_{\mathrm{jq}} \delta_{\mathrm{kr}}$

so that 


$$
\Delta \mathrm{E}=\frac{1}{2 \pi} \frac{16}{35} \frac{1}{\mathrm{R}^{-9}} \operatorname{Re} \int_{0}^{+\infty} d \omega \int_{0}^{+\infty} d \omega^{\prime} \sum_{\mathrm{ijk}}\left(\begin{array}{c}
\beta_{\mathrm{ijk}}\left(-\omega^{\prime}-\omega, \omega^{\prime}, \omega\right)_{\mathrm{B}} \beta_{\mathrm{ijk}}\left(\omega^{\prime}+\omega,-\omega^{\prime},-\omega_{\mathrm{A}}+\right. \\
\beta_{\mathrm{ijk}}\left(\omega^{\prime}+\omega,-\omega^{\prime},-\omega\right)_{\mathrm{B}} \beta_{\mathrm{ijk}}\left(-\omega^{\prime}-\omega^{\prime}, \omega^{\prime}, \omega\right)_{\mathrm{A}}
\end{array}\right)
$$

The integrals over the frequencies can be easily calculated by using

$$
\frac{1}{\omega-\omega_{0} \pm \mathrm{i} \varepsilon}=\mathrm{PP} \frac{1}{\omega-\omega_{0}} \mp \mathrm{i} \pi \delta\left(\omega-\omega_{0}\right)
$$

It appears that the real and imaginary contributions of most of the 72 terms cancel each other 2 by 2 ; actually only 4 terms described by the diagrams of fig. 3 have a non vanishing contribution (Appendix 3).

Finally we have

$$
\Delta E=\frac{16}{35} \frac{1}{R^{9}} \frac{1}{\left(4 \pi \epsilon_{0}\right)^{3}} \sum_{i j k} \sum_{m, n} \sum_{m^{\prime}, n^{\prime}} \frac{\left\langle 0\left|d_{i} P_{m} \cdot d_{j} P_{m} d_{k}\right| 0\right\rangle\left\langle 0\left|d_{i} P_{n^{\prime}} d_{j} P_{n} d_{k}\right| 0\right\rangle}{\left(2 E_{0}-E_{m^{\prime}}-E_{n^{\prime}}\right)\left(2 E_{0}-E_{m}-E_{n}\right)}
$$

It is easily verified that the resulting expression is the static term proposed in equ.(2) after averaging over molecular orientations. That interaction is always attractive and tends to align the molecules.

\section{Possibility of an helicoidal ordering}

$\beta^{(1)}$ and $\beta^{(3)}$ behave in a rotation like the irreducible unit tensors [7] $\mathrm{C}^{(1)}(\theta, \varphi)$ and $\mathrm{C}^{(3)}(\theta, \varphi)$ whereas $\alpha^{(2)}$ and $\eta^{(2)}$ behave like $\mathrm{C}^{(2)}(\theta, \varphi)$. Let $\theta_{\mathrm{A}}, \varphi_{\mathrm{A}}\left(\operatorname{resp} \theta_{\mathrm{B}}, \varphi_{\mathrm{B}}\right)$ be the polar angles of a molecular axis $\mathrm{Z}_{\mathrm{A}}\left(\right.$ resp. $\left.\mathrm{Z}_{\mathrm{B}}\right)$ in some external reference frame and let $\rho_{\mathrm{AB}}$ be the angle between $\mathrm{Z}_{\mathrm{A}}$ and $\mathrm{Z}_{\mathrm{B}}$. The interaction energy between the 2 molecules $\mathrm{A}$ and $\mathrm{B}$ can be written as

$\mathrm{U}_{\mathrm{AB}}=E_{1}(\mathrm{R}) \mathrm{P}_{1}\left(\cos \rho_{\mathrm{AB}}\right)+E_{2}(\mathrm{R}) \mathrm{P}_{2}\left(\cos \rho_{\mathrm{AB}}\right)+E_{3}(\mathrm{R}) \mathrm{P}_{3}\left(\cos \rho_{\mathrm{AB}}\right)$

$\mathrm{P}_{\ell}\left(\cos \rho_{\mathrm{AB}}\right)$ is the Legendre polynomial of order $\ell$ 
with $\mathrm{P}_{\ell}\left(\cos \rho_{\mathrm{AB}}\right)=\mathrm{C}^{(\ell)}\left(\theta_{\mathrm{A}}, \varphi_{\mathrm{A}}\right) \cdot \mathrm{C}^{(\ell)}\left(\theta_{\mathrm{B}}, \varphi_{\mathrm{B}}\right)$

and $\cos \rho_{\mathrm{AB}}=\cos \theta_{\mathrm{A}} \cos \theta_{\mathrm{B}}+\sin \theta_{\mathrm{A}} \sin \theta_{\mathrm{B}} \cos \left(\varphi_{\mathrm{A}}-\varphi_{\mathrm{B}}\right)$

It is worth recalling here that in the situation considered in ref.[3] the helicoidal ordering derives from the conflict between the quadrupolar Van der Waals interaction and the quadrupolar chiral interaction. Both interactions have the same symmetry properties with respect to rotations, and different ranges, but the Van der Waals term is always attractive and tends to align the molecules parallel or anti-parallel to one of the main axes of $\alpha^{(2)}$, conversely the chiral term is always repulsive and tends to align the molecules perpendicular to one of the main axes of $\eta^{(2)}$. The 2 couplings thus tend to align the molecules along two different directions and the system may minimise its free energy by adopting an helical ordering.

In the present situation, the molecular coupling associated with the nonlinear polarizabilities has a different symmetry property with respect to rotations (and also a different range) and that conflict could also cause an helicoidal ordering (the situation is somehow similar to the helimagnetic case).

The molecular planes $\Pi_{\mathrm{A}}$ and $\Pi_{\mathrm{B}}$ intersect each other along a direction which we choose as the $\mathrm{Z}$ axis. Let $\Phi$ be the angle between $\Pi_{\mathrm{A}}$ and $\Pi_{\mathrm{B}}$ (fig. 4) so that $\Phi=\varphi_{A}-\varphi_{B}$. Writing $\left(\frac{\partial \mathrm{U}}{\partial \Phi}\right)_{\mathrm{R}}=0$, the minima of $\mathrm{U}$ for any given value of $\mathrm{R}$ are the roots of $\sin \rho_{A B}=0$ i.e. $\rho_{A B}=0$ or $\rho_{A B}=\pi ; \rho_{A B}=0$ means $\Phi=0, \theta_{\mathrm{A}}=\theta_{\mathrm{B}}$, i.e. a parallel configuration, and it corresponds to a minimum of $\mathrm{U}$ whereas $\rho_{A B}=\pi$ means 
$\Phi=\pi, \theta_{\mathrm{B}}=-\theta_{\mathrm{A}}$ and corresponds to a maximum of $\mathrm{U}$. In the parallel configuration, $\Pi_{\mathrm{A}}$ and $\Pi_{\mathrm{B}}$ are identical and in that plane the molecules present a nematic-type ordering. If $\Pi_{\mathrm{A}}$ and $\Pi_{\mathrm{B}}$ are parallel, the $\mathrm{Z}$ axis as chosen above (intersection of $\Pi_{\mathrm{A}}$ and $\Pi_{\mathrm{B}}$ ) does not exist and we adopt a different approach. Let $\mathrm{Z}$ now be the axis perpendicular to $\Pi_{\text {A }}$ and $\Pi_{\text {B }}$ and let $\Theta$ be the angle between $\mathrm{Z}$ and $\mathbf{R}$ (fig. 5).

We look for the possibility of helical ordering by putting $\mathrm{t}=\cos \rho_{\mathrm{AB}}, \rho_{\mathrm{AB}}=\mathrm{qZ}, \mathrm{Z}=\mathrm{R} \cos \Theta$

At short distance, the molecules feel a strong repulsive interaction due to the exclusion principle; as they are supposed to be able to rotate freely and as their centres are assumed to be randomly distributed, the repulsive forces will be accounted for by an effective hard-core inter-molecular potential of radius D (effective molecular diameter).

We will express the quadrupole-type interaction as

$E_{2}(R)=\left\langle E_{V d W}\right\rangle\left(\frac{D}{R}\right)^{6}-\left\langle E_{\eta}\right\rangle\left(\frac{D}{R}\right)^{8}$ for $R>D$,

where the first term accounts for the anisotropic Van der Waals interaction and the second term for the interaction associated to the optical activity, the vector-type coupling associated to the nonlinear polarizability as $E_{1}(R)=\left\langle E_{1}\right\rangle\left(\frac{D}{R}\right)^{9}$ for $R>D$, and the octupole-type coupling associated to the nonlinear polarizability as $\mathrm{E}_{3}(\mathrm{R})=\left\langle\mathrm{E}_{3}\right\rangle\left(\frac{\mathrm{D}}{\mathrm{R}}\right)^{9}$ for $\mathrm{R}>\mathrm{D}$

The average interaction energy of 2 molecules A and B can thus be written as 


$$
\begin{aligned}
\left\langle\mathrm{U}_{\mathrm{AB}}\right\rangle= & \int \frac{d^{3} \mathbf{R}_{\mathrm{AB}}}{\mathrm{V}} \mathrm{Y}(\mathrm{R}-\mathrm{D}) \mathrm{U}_{\mathrm{AB}}(\mathrm{R}) \\
& =\left\langle-\mathrm{H}_{0}\right\rangle+\int \frac{\mathrm{R}^{2} d \mathrm{R}}{\mathrm{V}} \mathrm{Y}(\mathrm{R}-\mathrm{D})\left[\left\langle\mathrm{E}_{\mathrm{VdW}}\right\rangle\left(\frac{\mathrm{D}}{\mathrm{R}}\right)^{6}-\left\langle\mathrm{E}_{\eta}\right\rangle\left(\frac{\mathrm{D}}{\mathrm{R}}\right)^{8}\right] \int 2 \pi \sin \Theta d \Theta \mathrm{P}_{2}\left(\cos \rho_{\mathrm{AB}}\right) \\
& +\int \frac{\mathrm{R}^{2} d \mathrm{R}}{\mathrm{V}} \mathrm{Y}(\mathrm{R}-\mathrm{D})\left[\left\langle\mathrm{E}_{1}\right\rangle\left(\frac{\mathrm{D}}{\mathrm{R}}\right)^{9}\right] \int 2 \pi \sin \Theta d \Theta \mathrm{P}_{1}\left(\cos \rho_{\mathrm{AB}}\right) \\
& +\int \frac{\mathrm{R}^{2} d \mathrm{R}}{\mathrm{V}} \mathrm{Y}(\mathrm{R}-\mathrm{D})+\left[\left\langle\mathrm{E}_{3}\right\rangle\left(\frac{\mathrm{D}}{\mathrm{R}}\right)^{9}\right] \int 2 \pi \sin \Theta d \Theta \mathrm{P}_{3}\left(\cos \rho_{\mathrm{AB}}\right)
\end{aligned}
$$

where $Y(R-D)=0$ if $R<D$ and $Y(R-D)=1$ if $R>D$. Writing

$$
\begin{aligned}
& \mathrm{P}_{1}\left(\cos \rho_{\mathrm{AB}}\right)=\cos \rho_{\mathrm{AB}}=\operatorname{Re}(\operatorname{expiqR} \cos \Theta) \\
& \mathrm{P}_{2}\left(\cos \rho_{\mathrm{AB}}\right)=\frac{3 \cos ^{2} \rho_{\mathrm{AB}}-1}{2}=\frac{1}{4}+\frac{3}{4} \cos 2 \rho_{\mathrm{AB}}=\frac{1}{4}+\frac{3}{4} \operatorname{Re}(\exp 2 \mathrm{iqR} \cos \Theta) \\
& \mathrm{P}_{3}\left(\cos \rho_{\mathrm{AB}}\right)=\frac{5 \cos \rho_{\mathrm{AB}}^{3}-3 \cos \rho_{\mathrm{AB}}}{2}=\frac{5}{8} \cos 3 \rho_{\mathrm{AB}}+\frac{3}{8} \cos \rho_{\mathrm{AB}} \\
& =\frac{5}{8} \operatorname{Re}(\exp 3 i q \mathrm{R} \cos \Theta)+\frac{3}{8} \operatorname{Re}(\operatorname{expiqR} \cos \Theta)
\end{aligned}
$$

we have

$$
\begin{aligned}
\left\langle\mathrm{U}_{\mathrm{AB}}\right\rangle= & \left\langle-\mathrm{H}_{0}\right\rangle+\int_{\mathrm{D}}^{\infty} \frac{4 \pi \mathrm{R}^{2} d \mathrm{R}}{\mathrm{V}}\left[\left\langle\mathrm{E}_{\mathrm{VdW}}\right\rangle\left(\frac{\mathrm{D}}{\mathrm{R}}\right)^{6}-\left\langle\mathrm{E}_{\mathrm{\eta}}\right\rangle\left(\frac{\mathrm{D}}{\mathrm{R}}\right)^{8}\right]\left[\frac{1}{4}+\frac{3}{4} \frac{\sin 2 \mathrm{qR}}{2 \mathrm{qR}}\right] \\
& +\int_{\mathrm{D}}^{\infty} \frac{4 \pi \mathrm{R}^{2} d \mathrm{R}}{\mathrm{V}}\left[\left\langle\mathrm{E}_{1}\right\rangle\left(\frac{\mathrm{D}}{\mathrm{R}}\right)^{9}\right]\left[\frac{\sin \mathrm{qR}}{\mathrm{qR}}\right] \\
& +\int_{\mathrm{D}}^{\infty} \frac{4 \pi \mathrm{R}^{2} d \mathrm{R}}{\mathrm{V}}\left[\left\langle\mathrm{E}_{3}\right\rangle\left(\frac{\mathrm{D}}{\mathrm{R}}\right)^{9}\right]\left[\frac{5}{8} \frac{\sin 3 \mathrm{qR}}{3 \mathrm{qR}}+\frac{3}{8} \frac{\sin \mathrm{qR}}{\mathrm{qR}}\right]
\end{aligned}
$$

The minimum of $\left\langle U_{A B}\right\rangle$ is obtained from $\frac{\partial\left\langle U_{A B}\right\rangle}{\partial q}=0$, i.e. 


$$
\begin{aligned}
0=\frac{3}{2}\left\langle\mathrm{E}_{\mathrm{VdW}}\right\rangle \int_{\mathrm{D}}^{\infty} \frac{d \mathrm{R}}{\mathrm{R}^{3}}\left[\frac{2 \mathrm{qR} \cos 2 \mathrm{qR}-\sin 2 \mathrm{qR}}{(2 \mathrm{qR})^{2}}\right]-\frac{3}{2}\left\langle\mathrm{E}_{\mathrm{\eta}}\right\rangle \mathrm{D}^{2} \int_{\mathrm{D}}^{\infty} \frac{d \mathrm{R}}{\mathrm{R}^{5}}\left[\frac{2 \mathrm{qR} \cos 2 \mathrm{qR}-\sin 2 \mathrm{qR}}{(2 \mathrm{qR})^{2}}\right] \\
+\left[\left\langle\mathrm{E}_{1}\right\rangle+\frac{3}{8}\left\langle\mathrm{E}_{3}\right\rangle\right] \mathrm{D}^{3} \int_{\mathrm{D}}^{\infty} \frac{d \mathrm{R}}{\mathrm{R}^{6}}\left[\frac{\mathrm{qR} \cos \mathrm{qR}-\sin \mathrm{qR}}{(\mathrm{qR})^{2}}\right]+\frac{15}{8}\left\langle\mathrm{E}_{3}\right\rangle \mathrm{D}^{3} \int_{\mathrm{D}}^{\infty} \frac{d \mathrm{R}}{\mathrm{R}^{6}}\left[\frac{3 \mathrm{qR} \cos 3 \mathrm{qR}-\sin 3 \mathrm{qR}}{(3 \mathrm{qR})^{2}}\right]
\end{aligned}
$$

or

$$
\begin{aligned}
0=6\left\langle\mathrm{E}_{\mathrm{VdW}}\right\rangle \int_{2 \mathrm{qD}}^{\infty} \mathrm{dt}\left[\frac{\mathrm{t} \cos \mathrm{t}-\sin \mathrm{t}}{\mathrm{t}^{5}}\right]-24\left\langle\mathrm{E}_{\mathrm{\eta}}\right\rangle(\mathrm{qD})^{2} \int_{2 \mathrm{qD}}^{\infty} \mathrm{dt}\left[\frac{\mathrm{t} \cos \mathrm{t}-\sin \mathrm{t}}{\mathrm{t}^{7}}\right] \\
+\left[\left\langle\mathrm{E}_{1}\right\rangle+\frac{3}{8}\left\langle\mathrm{E}_{3}\right\rangle\right](\mathrm{qD})^{3} \int_{\mathrm{qD}}^{\infty} \mathrm{dt}\left[\frac{\mathrm{t} \cos \mathrm{t}-\sin \mathrm{t}}{\mathrm{t}^{8}}\right]+\frac{5.3^{6}}{8}\left\langle\mathrm{E}_{3}\right\rangle(\mathrm{qD})^{3} \int_{3 \mathrm{qD}}^{\infty} \mathrm{dt}\left[\frac{\mathrm{t} \cos \mathrm{t}-\sin \mathrm{t}}{\mathrm{t}^{8}}\right]
\end{aligned}
$$

That allows to determine $\mathrm{q}$ and hence the pitch $\mathrm{L}$ of the helix as $\mathrm{L}=2 \pi / \mathrm{q}$. Assuming

$$
\begin{aligned}
& \mathrm{L}>>\mathrm{D} \text {, i.e. } \mathrm{qD}<<1 \text { we get } \\
& \begin{aligned}
0=\left\langle\mathrm{E}_{\mathrm{VdW}}\right\rangle\left(-\frac{1}{\mathrm{qD}}+\frac{3 \pi}{8}+\mathrm{o}(\mathrm{qD})\right)-\frac{1}{3}\left\langle\mathrm{E}_{\mathrm{\eta}}\right\rangle\left(-\frac{1}{\mathrm{qD}}+\mathrm{o}(\mathrm{qD})\right) \\
+\frac{1}{12}\left[\left\langle\mathrm{E}_{1}\right\rangle+\frac{3}{8}\left\langle\mathrm{E}_{3}\right\rangle\right]\left(-\frac{1}{\mathrm{qD}}+\mathrm{o}(\mathrm{qD})\right)+\frac{15}{32}\left\langle\mathrm{E}_{3}\right\rangle\left(-\frac{1}{\mathrm{qD}}+\mathrm{o}(\mathrm{qD})\right)
\end{aligned}
\end{aligned}
$$

and hence

$$
\mathrm{L} \square \frac{3 \pi^{2}}{4} \mathrm{D} \frac{\left\langle\mathrm{E}_{\mathrm{VdW}}\right\rangle}{\left\langle\mathrm{E}_{\mathrm{VdW}}\right\rangle-\frac{1}{3}\left\langle\mathrm{E}_{\eta}\right\rangle+\frac{1}{12}\left\langle\mathrm{E}_{1}\right\rangle+\frac{1}{2}\left\langle\mathrm{E}_{3}\right\rangle}
$$

That pitch does not depend upon the temperature as we have performed a simple geometrical averaging; a thermodynamical averaging would have provided a temperature-dependent pitch.

\section{Discussion}


In terms of orders of magnitude, assuming an effective electric charge $\zeta e$ per molecule and an average gap $\hbar \Omega$, we have

$$
\begin{aligned}
& \alpha^{(2)} \square \frac{(\zeta e)^{2}\left\langle 3 z^{2}-r^{2}\right\rangle}{\hbar \Omega} \\
& \eta^{(2)} \square \frac{(\zeta e)^{2}\left\langle 6 z^{3}-3 x^{3}-3 y^{3}-r^{2}(2 z-x-y)\right\rangle}{\hbar \Omega} \\
& \beta^{(1)} \square \frac{(\zeta e)^{3}\left\langle z r^{2}\right\rangle}{(\hbar \Omega)^{2}} \\
& \beta^{(3)} \square \frac{(\zeta e)^{3}\left\langle 5 z^{3}-3 z\right\rangle}{(\hbar \Omega)^{2}}
\end{aligned}
$$

$E_{1}$ and $E_{3}$ are given by an integral over 2 frequencies, $E_{V d W}$ and $E_{\eta}$ by an integral over a single frequency. $\Delta / \hbar$ being an effective integration range for the frequencies, we have the following orders of magnitude

$$
\begin{aligned}
& E_{1} \square\left(\frac{\zeta^{2} \mathrm{e}^{2}}{4 \pi \epsilon_{0}}\right)^{3}\left(\frac{\left\langle\mathrm{r}^{3}\right\rangle}{\mathrm{R}^{3}}\right)^{2}\left(\frac{\Delta}{\hbar \Omega}\right)^{2}\left(\frac{1}{\hbar \Omega}\right)^{2} \frac{1}{\mathrm{R}^{3}} \\
& E_{3} \square\left(\frac{\zeta^{2} \mathrm{e}^{2}}{4 \pi \epsilon_{0}}\right)^{3}\left(\frac{\left\langle\mathrm{r}^{3}\right\rangle}{\mathrm{R}^{3}}\right)^{2}\left(\frac{\Delta}{\hbar \Omega}\right)^{2}\left(\frac{1}{\hbar \Omega}\right)^{2} \frac{1}{\mathrm{R}^{3}} \\
& E_{\mathrm{VdW}} \square\left(\frac{\zeta^{2} \mathrm{e}^{2}}{4 \pi \epsilon_{0}}\right)^{2}\left(\frac{\left\langle\mathrm{r}^{2}\right\rangle}{\mathrm{R}^{2}}\right)^{2}\left(\frac{\Delta}{\hbar \Omega}\right) \frac{1}{\hbar \Omega} \frac{1}{\mathrm{R}^{2}} \\
& E_{\eta} \square\left(\frac{\zeta^{2} \mathrm{e}^{2}}{4 \pi \epsilon_{0}}\right)^{2}\left(\frac{\left\langle\mathrm{r}^{3}\right\rangle}{\mathrm{R}^{3}}\right)^{2}\left(\frac{\Delta}{\hbar \Omega}\right) \frac{1}{\hbar \Omega} \frac{1}{\mathrm{R}^{2}}
\end{aligned}
$$

and consequently the ratios 
$\frac{\mathrm{E}_{\eta}}{\mathrm{E}_{V d W}} \square\left(\frac{\left\langle r^{3}\right\rangle}{\mathrm{R}\left\langle r^{2}\right\rangle}\right)^{2}$

$\frac{\mathrm{E}_{1}}{\mathrm{E}_{V d W}} \square \frac{\mathrm{E}_{3}}{\mathrm{E}_{V d W}} \square\left(\frac{\zeta^{2} e^{2}}{4 \pi \epsilon_{0}}\right) \frac{1}{\mathrm{R}}\left(\frac{\Delta}{\hbar \Omega}\right) \frac{1}{\hbar \Omega}\left(\frac{\left\langle r^{3}\right\rangle}{\mathrm{R}\left\langle r^{2}\right\rangle}\right)^{2}$

$\frac{\mathrm{E}_{1}}{\mathrm{E}_{\eta}} \square \frac{\mathrm{E}_{3}}{\mathrm{E}_{\eta}} \square\left(\frac{\zeta^{2} e^{2}}{4 \pi \epsilon_{0}}\right) \frac{1}{\mathrm{R}}\left(\frac{\Delta}{\hbar \Omega}\right) \frac{1}{\hbar \Omega}$

For nearest neighbour molecules $\mathrm{R} \square \mathrm{D}, \mathrm{D} \square 10^{-9} \mathrm{~m}, \hbar \Omega \square \Delta \square 1-10 \mathrm{eV}$. With those values, it appears that the 3 types of coupling a priori may have the same order of magnitude and hence contribute to the ordering at comparable levels. If however we assume that the Van der Waals coupling is larger than the two chiral couplings, i.e. if the factor $\left(\frac{\left\langle r^{3}\right\rangle}{\mathrm{R}\left\langle r^{2}\right\rangle}\right)^{2}$ is actually small, those chiral couplings have similar magnitudes; thus none of them can be neglected and both contribute at a comparable level to a possible helical ordering. Such an helical ordering is actually observed in the twisted nematics (cholesteric) phases, but further study would be needed in order to precisely attribute their behaviour to the interactions we have highlighted.

\section{Conclusion}

By going beyond the Van der Waals interaction, we have evidenced two different interactions specific to chiral molecules. One can be accounted for by the exchange of 3 virtual photons of the electric dipole type; the resulting interaction potential involves the product of the first nonlinear polarizabilities of the molecules. The other one can be accounted for by the exchange of 2 virtual photons, one being electric dipolar and the 
other being electric quadrupolar or magnetic dipolar; the resulting interaction potential involves the product of the optical activities of the molecules. Both have in common to be third-order dispersion forces. Since they might have the same order of magnitude none can a priori be neglected and both might equally contribute to the possibility of an helical ordering. 


\section{Annexe 1}

In the Van der Waals interaction involving the exchange of 2 photons and 4 electricdipole couplings, the transition amplitude is given by

$$
\begin{aligned}
T_{i \rightarrow f}=T_{i \rightarrow f}^{0}+ & (2 \pi)^{-1}\left(t_{f}-t_{i}\right) \exp \left[-i E_{0}\left(t_{f}-t_{i}\right) \hbar^{-1}\right] \\
& \int_{0}^{+\infty} d \omega \sum_{i j p q} \alpha_{i j}(\omega)_{B} \alpha_{p q}(\omega)_{A} R^{-6} D_{i p}(i \omega R / c) D_{j q}(i \omega R / c)
\end{aligned}
$$

with

$$
\mathrm{D}_{\mathrm{j} 1}(\mathrm{x})=\delta_{\mathrm{j} 1}\left(1-\mathrm{x}+\mathrm{x}^{2}\right) \exp (\mathrm{x})-\frac{\mathrm{R}_{\mathrm{j}} \mathrm{R}_{1}}{\mathrm{R}^{2}}\left(3-3 \mathrm{x}+\mathrm{x}^{2}\right) \exp (\mathrm{x})
$$

Calling $\Delta \mathrm{E}$ the energy shift caused by the molecular interactions

$$
\mathrm{T}_{\mathrm{i} \rightarrow \mathrm{f}}=\exp \left[-\mathrm{i}\left(\mathrm{E}_{0}+\Delta \mathrm{E}\right)\left(\mathrm{t}_{\mathrm{f}}-\mathrm{t}_{\mathrm{i}}\right) \hbar^{-1}\right] \cong\left[1-\mathrm{i} \Delta \mathrm{E}\left(\mathrm{t}_{\mathrm{f}}-\mathrm{t}_{\mathrm{i}}\right) \hbar^{-1}\right] \exp \left[-\mathrm{iE}_{0}\left(\mathrm{t}_{\mathrm{f}}-\mathrm{t}_{\mathrm{i}}\right) \hbar^{-1}\right]
$$

we get

$$
\Delta \mathrm{E}=\frac{-1}{2 \pi} \operatorname{Im}\left(\int_{0}^{+\infty} \hbar d \omega \sum_{\mathrm{ijpq}} \alpha_{\mathrm{ij}}(\omega)_{\mathrm{B}} \alpha_{\mathrm{pq}}(\omega)_{\mathrm{A}} \mathrm{R}^{-6} \mathrm{D}_{\mathrm{ip}}(\mathrm{i} \omega \mathrm{R} / \mathrm{c}) \mathrm{D}_{\mathrm{jq}}(\mathrm{i} \omega \mathrm{R} / \mathrm{c})\right)
$$

If the retardation effects are neglected ( $\omega \mathrm{R} / \mathrm{c} \square 1$ ), then

$$
\mathrm{D}_{\text {ip }} \rightarrow \mathrm{T}_{\mathrm{ip}}=\delta_{\mathrm{ip}}-\frac{3 \mathrm{R}_{\mathrm{i}} \mathrm{R}_{\mathrm{p}}}{\mathrm{R}^{2}}
$$

i.e. $\mathrm{D}_{\mathrm{ip}}$ as a function of $\mathbf{R}$ is reduced to a pure quadrupole, independent of the frequency. The integrals over the frequencies can be easily calculated by using

$$
\frac{1}{\omega-\omega_{0} \pm \mathrm{i} \varepsilon}=\mathrm{PP} \frac{1}{\omega-\omega_{0}} \mp \mathrm{i} \pi \delta\left(\omega-\omega_{0}\right)
$$

and gives gain the classical expression

$$
\Delta \mathrm{E}=\frac{1}{\left(4 \pi \epsilon_{0}\right)^{2}} \sum_{\mathrm{ij}} \sum_{\mathrm{m}, \mathrm{n}} \frac{\left\langle 0_{\mathrm{A}} 0_{\mathrm{B}}\left|\mathrm{d}_{\mathrm{A}} \mathrm{T}_{\mathrm{AB}} \mathrm{d}_{\mathrm{B}}\right| \mathrm{m}_{\mathrm{A}} \mathrm{n}_{\mathrm{B}}\right\rangle\left\langle\mathrm{m}_{\mathrm{A}} \mathrm{n}_{\mathrm{B}}\left|\mathrm{d}_{\mathrm{A}} \mathrm{T}_{\mathrm{AB}} \mathrm{d}_{\mathrm{B}}\right| 0_{\mathrm{A}} 0_{\mathrm{B}}\right\rangle}{2 \mathrm{E}_{0}-\mathrm{E}_{\mathrm{m}}-\mathrm{E}_{\mathrm{n}}}
$$


The scalar component $\left\{\mathrm{D}_{\mathrm{ik}} \mathrm{D}_{\mathrm{j} 1}\right\}^{(0)}$ of $\mathrm{D}_{\mathrm{ik}} \mathrm{D}_{\mathrm{jl}}$ is

$\left\{D_{i k} D_{j 1}\right\}^{(0)}=\delta_{i k} \delta_{j 1} \frac{2}{3}\left(3-6 x+5 x^{2}-2 x^{3}+x^{4}\right) \exp (2 x)$

with $x=i \omega R / c$.

In the quasi static limit, the scalar contribution of $\mathrm{D}_{\mathrm{ip}} \mathrm{D}_{\mathrm{jq}}$ is

$\mathrm{D}_{\mathrm{ip}} \mathrm{D}_{\mathrm{jq}} \rightarrow \frac{4}{5} \delta_{\mathrm{ip}} \delta_{\mathrm{jq}}$

so that

$$
\Delta \mathrm{E}=\frac{-1}{2 \pi} \operatorname{Im}\left(\frac{4}{5 \mathrm{R}^{6}} \int_{0}^{+\infty} \mathrm{d} \omega \sum_{\mathrm{ij}} \alpha_{\mathrm{ij}}(\omega)_{\mathrm{B}} \alpha_{\mathrm{ij}}(\omega)_{\mathrm{A}}\right)
$$

which is equivalent to

$$
\Delta E=\frac{4}{5 R^{6}} \frac{1}{\left(4 \pi \epsilon_{0}\right)^{2}} \sum_{i j} \sum_{m, n} \frac{\left\langle 0\left|d_{i} P_{m} d_{j}\right| 0\right\rangle\left\langle 0\left|d_{i} P_{m} d_{j}\right| 0\right\rangle}{2 E_{0}-E_{m}-E_{n}}
$$

In the long distance limit, equ.(A1.4) is approximated by

$$
\Delta \mathrm{E} \square \frac{-1}{2 \pi} \operatorname{Im}\left(\sum_{\mathrm{ijpq}} \alpha_{\mathrm{ij}}(0)_{\mathrm{B}} \alpha_{\mathrm{pq}}(0)_{\mathrm{A}} \mathrm{R}^{-6} \int_{0}^{+\infty} \hbar d \omega \mathrm{D}_{\mathrm{ip}}(\mathrm{i} \omega \mathrm{R} / \mathrm{c}) \mathrm{D}_{\mathrm{jq}}(\mathrm{i} \omega \mathrm{R} / \mathrm{c})\right)
$$

The scalar contribution of $\mathrm{D}_{\mathrm{ip}} \mathrm{D}_{\mathrm{jq}}$ can be checked to be equal to

$$
\frac{2}{3}\left(3-6 x+5 x^{2}-2 x^{3}+x^{4}\right) \exp (2 x)
$$

so that

$$
\Delta \mathrm{E} \square \frac{-1}{2 \pi} \operatorname{Im}\left(\sum_{\mathrm{ijpq}} \alpha_{\mathrm{ij}}(0)_{\mathrm{B}} \alpha_{\mathrm{ij}}(0)_{\mathrm{A}} \mathrm{R}^{-6} \int_{0}^{+\infty} \hbar d \omega \frac{2}{3}\left(3-6 \mathrm{x}+5 \mathrm{x}^{2}-2 \mathrm{x}^{3}+\mathrm{x}^{4}\right) \exp (2 \mathrm{x})\right)
$$

The integral can be exactly calculated by putting $\xi=-\mathrm{i} \omega / \mathrm{R} / \mathrm{c}$ and an integration over the contour of fig.(3) and it finally leads to 


$$
\Delta \mathrm{E}=\frac{23 \hbar \mathrm{c}}{12 \pi}\left(\sum_{\mathrm{ijpq}} \alpha_{\mathrm{ij}}(0)_{\mathrm{B}} \alpha_{\mathrm{ij}}(0)_{\mathrm{A}} \mathrm{R}^{-7}\right)
$$

1

2

3

5

6

7

8

9

10

11

12

13

14

15

16

17

18

19

20

21

22

23

24

25

26

27

28

29

30

31

32

33

34

35

36

37

38

39

40

41

42

43

44

45

46

47

48

49

50

51

52

53

54

55

56

57

58

59

60 
Annexe 2

The transition amplitude for the 1st diagram of fig.(3) is

$$
\begin{aligned}
& \mathrm{T}_{\mathrm{i} \rightarrow \mathrm{f}}=\lim _{\varepsilon, \varepsilon^{\prime}, \varepsilon^{\prime \prime}, \varepsilon_{\mathrm{A}}, \varepsilon_{\mathrm{A}}^{\prime}, \varepsilon_{\mathrm{B}}, \varepsilon_{\mathrm{B}}^{\prime} \rightarrow 0} \int_{-\infty}^{+\infty} d \mathrm{t}_{6} \int_{-\infty}^{+\infty} d \mathrm{t}_{5} \int_{-\infty}^{+\infty} d \mathrm{t}_{4} \int_{-\infty}^{+\infty} d \mathrm{t}_{3} \int_{-\infty}^{+\infty} d \mathrm{t}_{2} \int_{-\infty}^{+\infty} d \mathrm{t}_{1} \\
& (\mathrm{i} / 2 \pi)^{7} \int d \mathrm{E}_{\mathrm{A}}^{\prime} \int d \mathrm{E}_{\mathrm{A}} \int d \mathrm{E}^{\prime}{ }_{\mathrm{B}} \int d \mathrm{E}_{\mathrm{B}} \int d\left(\omega^{\prime \prime 2}\right) \int d\left(\omega^{\prime 2}\right) \int d\left(\omega^{2}\right) \\
& \exp -\mathrm{i}\left(\mathrm{t}_{\mathrm{f}}-\mathrm{t}_{3}\right) \mathrm{E}_{0} \hbar^{-1} \cdot \exp -\mathrm{i}\left(\mathrm{t}_{3}-\mathrm{t}_{2}\right) \mathrm{E}_{\mathrm{A}}^{\prime} \hbar^{-1} \cdot \exp -\mathrm{i}\left(\mathrm{t}_{2}-\mathrm{t}_{1}\right) \mathrm{E}_{\mathrm{A}} \hbar^{-1} \cdot \exp -\mathrm{i}\left(\mathrm{t}_{1}-\mathrm{t}_{\mathrm{i}}\right) \mathrm{E}_{0} \hbar^{-1} \\
& \exp -\mathrm{i}\left(\mathrm{t}_{\mathrm{f}}-\mathrm{t}_{6}\right) \mathrm{E}_{0} \hbar^{-1} \cdot \exp -\mathrm{i}\left(\mathrm{t}_{6}-\mathrm{t}_{5}\right) \mathrm{E}_{\mathrm{B}}^{\prime} \hbar^{-1} \cdot \exp -\mathrm{i}\left(\mathrm{t}_{5}-\mathrm{t}_{4}\right) \mathrm{E}_{\mathrm{B}} \hbar^{-1} \cdot \exp -\mathrm{i}\left(\mathrm{t}_{4}-\mathrm{t}_{\mathrm{i}}\right) \mathrm{E}_{0} \hbar^{-1}
\end{aligned}
$$

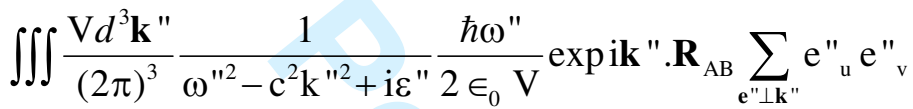

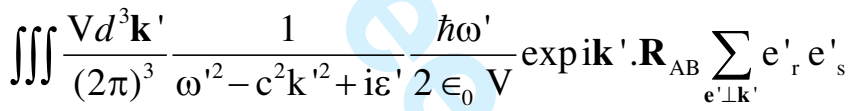

$$
\begin{aligned}
& \iiint \frac{\mathrm{V} d^{3} \mathbf{k}}{(2 \pi)^{3}} \frac{1}{\omega^{2}-\mathrm{c}^{2} \mathrm{k}^{2}+\mathrm{i} \varepsilon} \frac{\hbar \omega}{2 \in_{0} \mathrm{~V}} \exp -\mathrm{ik} \cdot \mathbf{R}_{\mathrm{AB}} \sum_{\mathbf{e} \perp \mathbf{k}} \mathrm{e}_{\mathrm{p}} \mathrm{e}_{\mathrm{q}} \\
& \sum_{n, n^{\prime}} \frac{\left\langle 0\left|d_{v}(B)\right| n^{\prime}\right\rangle\left\langle n^{\prime}\left|d_{s}(B)\right| n\right\rangle\left\langle n\left|d_{q}(B)\right| 0\right\rangle}{\left(E_{B}^{\prime}-\hbar \omega^{\prime \prime}+i \varepsilon_{B}^{\prime}\right)\left(E_{0}-E_{B}-\hbar \omega+i \varepsilon_{B}\right)} \\
& \sum_{\mathrm{m}, \mathrm{m}^{\prime}} \frac{\left\langle 0\left|\mathrm{~d}_{\mathrm{u}}(\mathrm{A})\right| \mathrm{n}^{\prime}\right\rangle\left\langle\mathrm{n}^{\prime}\left|\mathrm{d}_{\mathrm{r}}(\mathrm{A})\right| \mathrm{n}\right\rangle\left\langle\mathrm{n}\left|\mathrm{d}_{\mathrm{p}}(\mathrm{A})\right| 0\right\rangle}{\left.{ }_{\mathrm{A}}+\hbar \omega^{\prime \prime}+\mathrm{i} \varepsilon_{\mathrm{A}}^{\prime}\right)\left(\mathrm{E}_{0}-\mathrm{E}_{\mathrm{A}}+\hbar \omega+\mathrm{i} \varepsilon_{\mathrm{A}}\right)}
\end{aligned}
$$

The other diagrams can be expressed in a similar way. We get

$$
\begin{aligned}
& \mathrm{T}_{\mathrm{i} \rightarrow \mathrm{f}}=\lim _{\varepsilon, \varepsilon^{\prime}, \varepsilon^{\prime \prime} \rightarrow 0}(-\mathrm{i})\left(\hbar / 4 \pi^{2}\right)\left(\mathrm{t}_{\mathrm{f}}-\mathrm{t}_{\mathrm{i}}\right) \exp \left[-\mathrm{iE}_{0}\left(\mathrm{t}_{\mathrm{f}}-\mathrm{t}_{\mathrm{i}}\right) \hbar^{-1}\right] \\
& \int_{0}^{\infty} \omega^{\prime \prime 2} d \omega^{\prime \prime} \int_{0}^{\infty} \omega^{\prime 2} d \omega^{\prime} \int_{0}^{\infty} \omega^{2} d \omega \quad \delta\left(\omega^{\prime \prime}-\omega^{\prime}-\omega\right) \\
& \left.\epsilon_{0}^{-3} \sum_{n, n^{\prime}} \frac{\left\langle 0\left|\mathrm{~d}_{\mathrm{v}}(\mathrm{B})\right| \mathrm{n}^{\prime}\right\rangle\left\langle\mathrm{n}^{\prime}\left|\mathrm{d}_{\mathrm{s}}(\mathrm{B})\right| \mathrm{n}\right\rangle\left\langle\mathrm{n}\left|\mathrm{d}_{\mathrm{q}}(\mathrm{B})\right| 0\right\rangle}{\left(\mathrm{n}^{\prime}\right.}-\hbar \omega^{\prime \prime}+\mathrm{i} \varepsilon_{\mathrm{B}}^{\prime}\right)\left(\mathrm{E}_{0}-\mathrm{E}_{\mathrm{n}}-\hbar \omega+\mathrm{i} \varepsilon_{\mathrm{B}}\right) \sum_{\mathrm{m}, \mathrm{m}^{\prime}} \frac{\left\langle 0\left|\mathrm{~d}_{\mathrm{u}}(\mathrm{A})\right| \mathrm{n}^{\prime}\right\rangle\left\langle\mathrm{n}^{\prime}\left|\mathrm{d}_{\mathrm{r}}(\mathrm{A})\right| \mathrm{n}\right\rangle\left\langle\mathrm{n}\left|\mathrm{d}_{\mathrm{p}}(\mathrm{A})\right| 0\right\rangle}{\left(\mathrm{E}_{\mathrm{n}^{\prime}}+\hbar \omega^{\prime \prime}+\mathrm{i} \varepsilon_{\mathrm{A}}{ }_{\mathrm{A}}\right)\left(\mathrm{E}_{0}-\mathrm{E}_{\mathrm{n}}+\hbar \omega+\mathrm{i} \varepsilon_{\mathrm{A}}\right)} \\
& \iiint \frac{d^{3} \mathbf{k} "}{(2 \pi)^{3}} \frac{1}{\omega^{\prime \prime 2}-c^{2} k^{\prime 2}+\mathrm{i} \varepsilon^{\prime \prime}} \iiint \frac{d^{3} \mathbf{k}^{\prime}}{(2 \pi)^{3}} \frac{1}{\omega^{\prime 2}-c^{2} \mathbf{k}^{\prime 2}+\mathrm{i} \varepsilon^{\prime}} \iiint \frac{d^{3} \mathbf{k}}{(2 \pi)^{3}} \frac{1}{\omega^{2}-\mathrm{c}^{2} \mathrm{k}^{2}+\mathrm{i} \varepsilon} \\
& \operatorname{expi}\left(\mathbf{k}+\mathbf{k}^{\prime}-\mathbf{k}^{\prime \prime}\right) \cdot \mathbf{R}_{\mathrm{AB}} \sum_{\mathbf{e}^{\prime \prime} \perp \mathbf{k}^{\prime \prime}} \mathrm{e}^{\prime \prime} \mathrm{e}{ }^{\prime \prime} \sum_{\mathbf{e}^{\prime} \perp \mathbf{k}^{\prime}} \mathrm{e}_{\mathrm{r}} \mathrm{e}_{\mathrm{s}}^{\prime} \sum_{\mathbf{e} \perp \mathbf{k}} \mathrm{e}_{\mathrm{p}} \mathrm{e}_{\mathrm{q}}
\end{aligned}
$$


The total transition amplitude is $T_{i \rightarrow f}=T_{i \rightarrow f}^{0}+T_{i \rightarrow f}$ where $\mathrm{T}_{\mathrm{i} \rightarrow \mathrm{f}}^{0}=\exp \left[-\mathrm{iE}_{0}\left(\mathrm{t}_{\mathrm{f}}-\mathrm{t}_{\mathrm{i}}\right) \hbar^{-1}\right]$ accounts for the trivial un-perturbated case.

It is easy to check that $\sum_{\mathbf{e} \perp \mathbf{k}} \mathrm{e}_{\mathrm{j}} \mathrm{e}_{1}=\delta_{\mathrm{jl}}-\frac{\mathrm{k}_{\mathrm{j}} \mathrm{k}_{1}}{\mathrm{k}^{2}}$.

Then we have to calculate the two integrals

$\lim _{\varepsilon \rightarrow 0} \iiint \frac{d^{3} \mathbf{k}}{(2 \pi)^{3}} \frac{1}{\omega^{2}-c^{2} k^{2}+i \varepsilon} \exp -i \mathbf{k} \cdot \mathbf{R}_{\mathrm{AB}}$

and

$\lim _{\varepsilon \rightarrow 0} \iiint \frac{\mathrm{d}^{3} \mathbf{k}}{(2 \pi)^{3}} \frac{\mathrm{k}_{\mathrm{j}} \mathrm{k}_{1}}{\mathrm{k}^{2}} \frac{1}{\omega^{2}-\mathrm{c}^{2} \mathrm{k}^{2}+\mathrm{i} \varepsilon} \exp -\mathrm{ik} \cdot \mathbf{R}_{\mathrm{AB}}$

The second integral is

$\lim _{\varepsilon \rightarrow 0^{-}}-\frac{\mathrm{c}^{2}}{\omega^{2}} \nabla_{\mathrm{j}} \nabla_{1} \iiint \frac{\mathrm{d}^{3} \mathbf{k}}{(2 \pi)^{3}} \frac{1}{\omega^{2}-\mathrm{c}^{2} \mathrm{k}^{2}+\mathrm{i} \varepsilon} \exp -\mathrm{ik} \cdot \mathrm{R}_{\mathrm{AB}}$

and the first integral is

$\lim _{\varepsilon \rightarrow 0} \frac{4 \pi}{(2 \pi)^{3}} \int_{-\infty}^{+\infty} \frac{k^{2} d k}{\omega^{2}-c^{2} k^{2}+i \varepsilon} \frac{\operatorname{expikR}}{2 i k R}=-\frac{\operatorname{expi\omega R} / c}{4 \pi c^{2} R}$

We finally obtain

$\lim _{\varepsilon \rightarrow 0} \iiint \frac{d^{3} \mathbf{k}}{(2 \pi)^{3}} \frac{1}{\omega^{2}-c^{2} \mathrm{k}^{2}+\mathrm{i} \varepsilon} \exp -\mathrm{ik} \cdot \mathbf{R}_{\mathrm{AB}} \sum_{\mathbf{e} \perp \mathbf{k}} \mathrm{e}_{\mathrm{j}} \mathrm{e}_{1}=-\left(\delta_{\mathrm{jl}}+\frac{\mathrm{c}^{2}}{\omega^{2}} \nabla_{\mathrm{j}} \nabla_{1}\right)\left(\frac{\operatorname{expi\omega R/c}}{4 \pi \mathrm{c}^{2} \mathrm{R}}\right)$
$==\delta_{\mathrm{j} 1}\left(1-\left(\frac{\mathrm{i} \omega \mathrm{R}}{\mathrm{c}}\right)+\left(\frac{\mathrm{i} \omega \mathrm{R}}{\mathrm{c}}\right)^{2}\right)\left(\frac{\operatorname{expi\omega R/c}}{4 \pi \omega^{2} \mathrm{R}^{3}}\right)-\frac{\mathrm{R}_{\mathrm{j}} \mathrm{R}_{1}}{\mathrm{R}^{2}}\left(3-3\left(\frac{\mathrm{i} \omega \mathrm{R}}{\mathrm{c}}\right)+\left(\frac{\mathrm{i} \omega \mathrm{R}}{\mathrm{c}}\right)^{2}\right)\left(\frac{\operatorname{expi\omega R/c}}{4 \pi \omega^{2} \mathrm{R}^{3}}\right)$

(A2.8)

Let us put 


$$
\begin{aligned}
D_{j 1}(x) & =\delta_{j 1}\left(1-x+x^{2}\right) \exp (x)-\frac{R_{j} R_{1}}{R^{2}}\left(3-3 x+x^{2}\right) \exp (x) \\
& =\delta_{j 1} \frac{2}{3} x^{2} \exp (x)-\left(\frac{3 R_{j} R_{1}-R^{2} \delta_{j 1}}{2 R^{2}}\right) \frac{2}{3}\left(3-3 x+x^{2}\right) \exp (x)
\end{aligned}
$$

We obtain

$$
\begin{aligned}
\mathrm{T}_{\mathrm{i} \rightarrow \mathrm{f}}= & \lim _{\varepsilon, \varepsilon^{\prime}, \varepsilon^{\prime \prime} \rightarrow 0}(-\mathrm{i})\left(\hbar / 4 \pi^{2}\right)\left(\mathrm{t}_{\mathrm{f}}-\mathrm{t}_{\mathrm{i}}\right) \exp \left[-\mathrm{iE}_{0}\left(\mathrm{t}_{\mathrm{f}}-\mathrm{t}_{\mathrm{i}}\right) \hbar^{-1}\right] \\
& \mathrm{R}^{-9} \int_{0}^{\infty} \omega^{\prime \prime 2} d \omega^{\prime \prime} \int_{0}^{\infty} \omega^{\prime 2} d \omega^{\prime} \int_{0}^{\infty} \omega^{2} d \omega \delta\left(\omega^{\prime \prime}-\omega^{\prime}-\omega\right) \mathrm{D}_{\mathrm{vu}}(\mathrm{x}) \mathrm{D}_{\mathrm{sr}}(\mathrm{x}) \mathrm{D}_{\mathrm{qp}}(\mathrm{x}) \\
& \epsilon_{0}^{-3} \sum_{\mathrm{n}, \mathrm{n}^{\prime}} \frac{\left\langle 0\left|\mathrm{~d}_{\mathrm{v}}(\mathrm{B})\right| \mathrm{n}^{\prime}\right\rangle\left\langle\mathrm{n}^{\prime}\left|\mathrm{d}_{\mathrm{s}}(\mathrm{B})\right| \mathrm{n}\right\rangle\left\langle\mathrm{n}\left|\mathrm{d}_{\mathrm{q}}(\mathrm{B})\right| 0\right\rangle}{\left(\mathrm{E}_{0}-\mathrm{E}_{\mathrm{n}^{\prime}}-\hbar \omega^{\prime \prime}+\mathrm{i}_{\mathrm{B}}^{\prime}\right)\left(\mathrm{E}_{0}-\mathrm{E}_{\mathrm{n}}-\hbar \omega+\mathrm{i} \varepsilon_{\mathrm{B}}\right)} \sum_{\mathrm{m}, \mathrm{m}^{\prime}} \frac{\left\langle 0\left|\mathrm{~d}_{\mathrm{u}}(\mathrm{A})\right| \mathrm{n}^{\prime}\right\rangle\left\langle\mathrm{n}^{\prime}\left|\mathrm{d}_{\mathrm{r}}(\mathrm{A})\right| \mathrm{n}\right\rangle\left\langle\mathrm{n}\left|\mathrm{d}_{\mathrm{p}}(\mathrm{A})\right| 0\right\rangle}{\left.\mathrm{E}_{\mathrm{n}^{\prime}}+\hbar \omega^{\prime \prime}+\mathrm{i} \varepsilon_{\mathrm{A}}^{\prime}\right)\left(\mathrm{E}_{0}-\mathrm{E}_{\mathrm{n}}+\hbar \omega+\mathrm{i} \varepsilon_{\mathrm{A}}\right)}
\end{aligned}
$$

(A2.10) 
Annexe 3

The 4 terms described by the diagrams of fig. 3 are resonant at the frequency $\omega$ and involve the integrals

$$
\begin{aligned}
& \left.\int_{0}^{\infty} \frac{d \omega}{\left(E_{0}-\right.} E_{n}+\hbar \omega+i \varepsilon\right)\left(E_{0}-E_{n^{\prime}}-\hbar \omega-i \varepsilon\right) \\
& \quad=\frac{1}{\left(E_{0}-E_{n^{\prime}}\right)+\left(E_{0}-E_{n}\right)} \int_{0}^{\infty} d \omega\left[\frac{1}{\left(E_{0}-E_{n}+\hbar \omega+i \varepsilon\right)}+\frac{1}{\left(E_{0}-E_{n^{\prime}}-\hbar \omega-i \varepsilon\right)}\right] \\
& =\frac{1}{\left(2 E_{0}-E_{n}-E_{n^{\prime}}\right)} \log \left(\frac{E_{0}-E_{n^{\prime}}}{E_{0}-E_{n}}\right)-\frac{i \pi}{\left(2 E_{0}-E_{n}-E_{n^{\prime}}\right)}
\end{aligned}
$$

But

$$
\sum_{n, n^{\prime}} \frac{1}{\left(2 E_{0}-E_{n}-E_{n^{\prime}}\right)} \log \left(\frac{E_{0}-E_{n^{\prime}}}{E_{0}-E_{n}}\right)=0
$$

so that

$$
\sum_{n, n^{\prime}} \int_{0}^{\infty} \frac{d \omega}{\left(E_{0}-E_{n}+\hbar \omega+i \varepsilon\right)\left(E_{0}-E_{n^{\prime}}-\hbar \omega-i \varepsilon\right)}=\frac{-i \pi}{\left(2 E_{0}-E_{n}-E_{n^{\prime}}\right)}
$$

Hence

$$
\begin{aligned}
\sum_{m, m^{\prime}, n, n^{\prime}} \int_{0}^{\infty} & \frac{d \omega}{\left(E_{0}-E_{n}+\hbar \omega+i \varepsilon\right)\left(E_{0}-E_{n^{\prime}}-\hbar \omega-i \varepsilon\right)} \int_{0}^{\infty} \frac{d \omega^{\prime}}{\left(E_{0}-E_{m}+\hbar \omega^{\prime}+i \varepsilon^{\prime}\right)\left(E_{0}-E_{m^{\prime}}-\hbar \omega^{\prime}-i \varepsilon^{\prime}\right)} \\
& =\sum_{m, m^{\prime}, n, n^{\prime}} \frac{-\pi^{2}}{\left(2 E_{0}-E_{n}-E_{n^{\prime}}\right)\left(2 E_{0}-E_{m}-E_{m^{\prime}}\right)}
\end{aligned}
$$




\section{Figure caption:}

Fig.1: Diagrams expressing the first order non linear optical phenomena (frequency sum and difference).

Fig.2: The 3 types of topologically different diagrams expressing the interaction of the 2 molecules A and B via the exchange of 3 virtual photons.

Fig.3: The 4 diagrams actually contributing to the interaction energy.

Fig.4: Relative configurations of the molecular planes: case of intersecting molecular planes.

Fig.5: Relative configurations of the molecular planes: case of parallel molecular planes. 
Fig.1

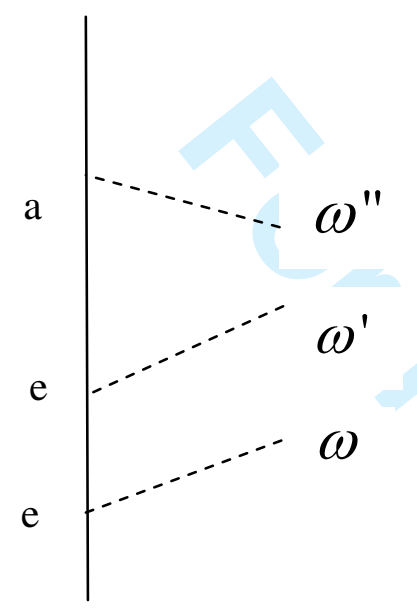

A

Fig. 1a

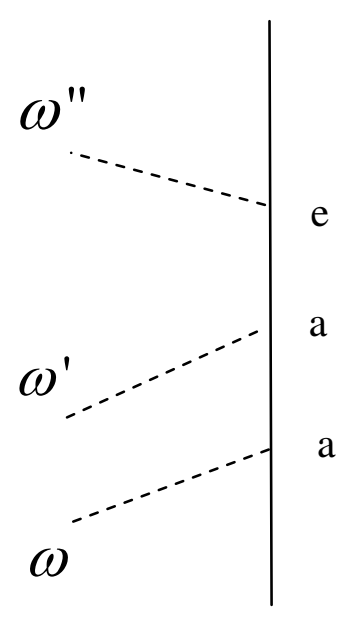

A

Fig. 1b

30

31

32

33

34

35

36

37

38

39

40

41

42

43

44

45

46

47

48

49

50

51

52

53

54

55

56

57

58

59

60 
Fig. 2

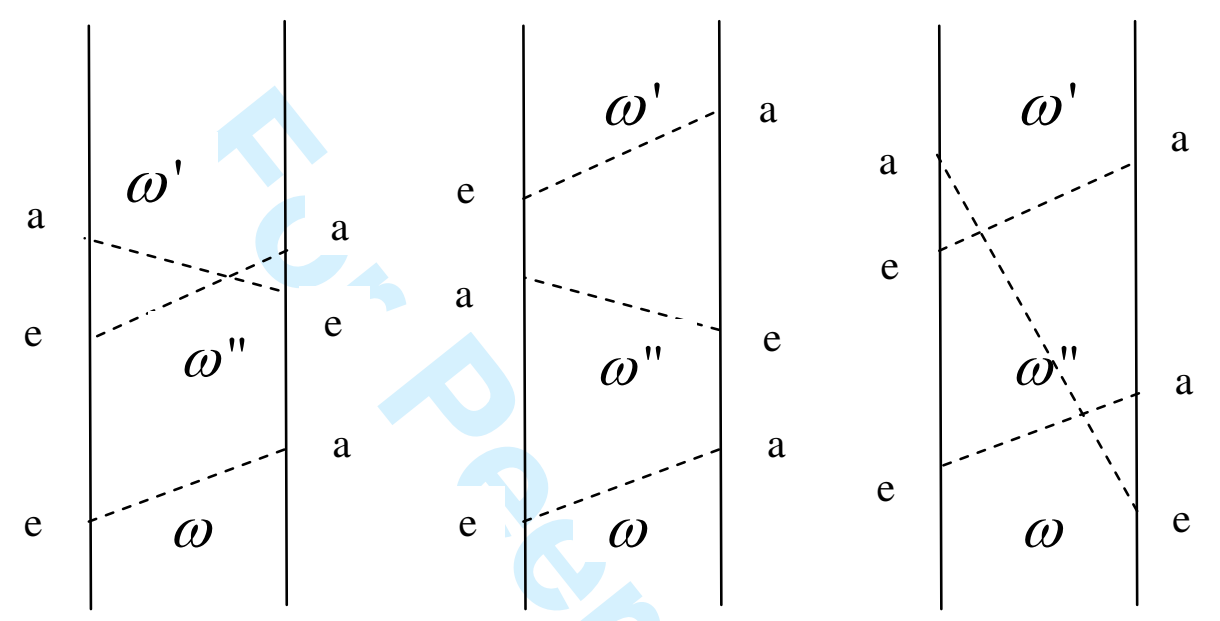


Fig.3

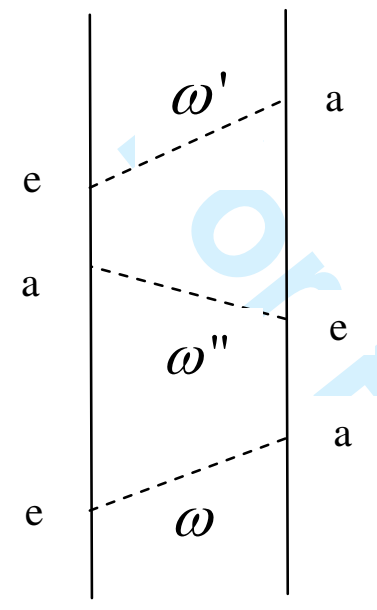

A $B$

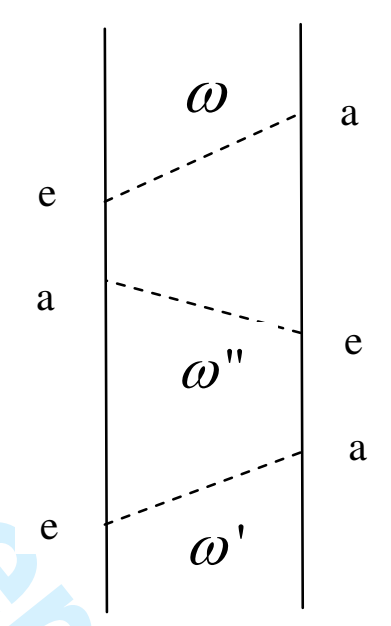

A $\quad$ B

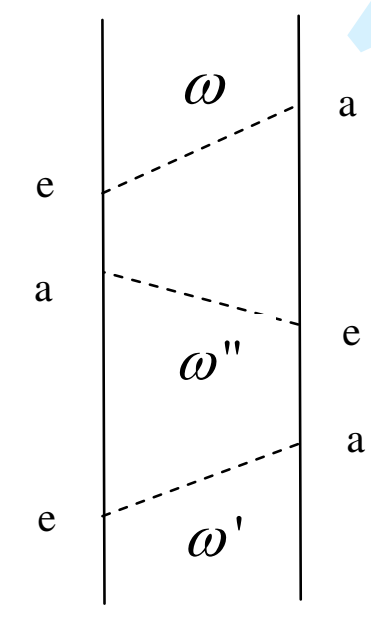

B

A 
Fig. 4

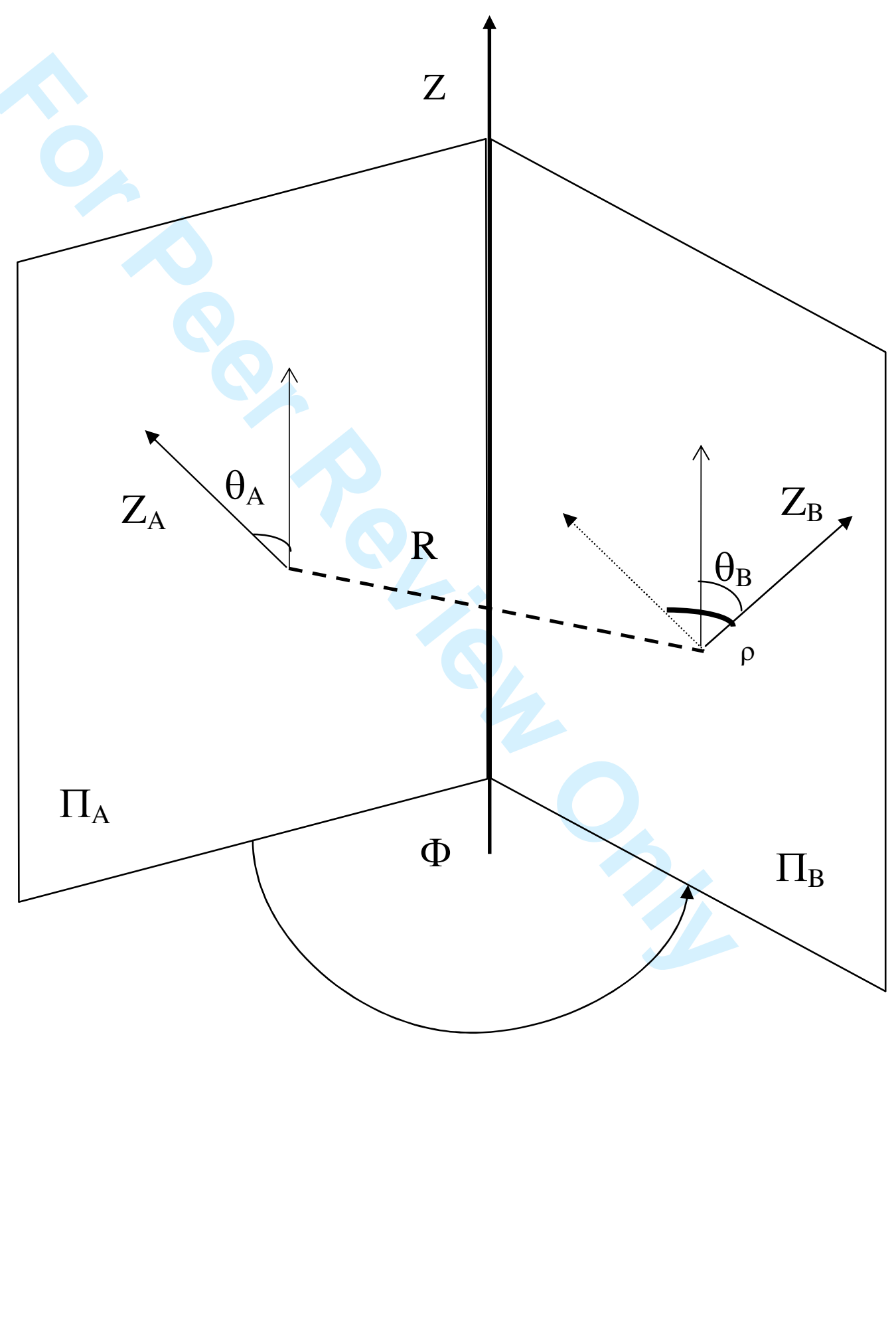

URL: http://mc.manuscriptcentral.com/tandf/tmph 
Fig. 5

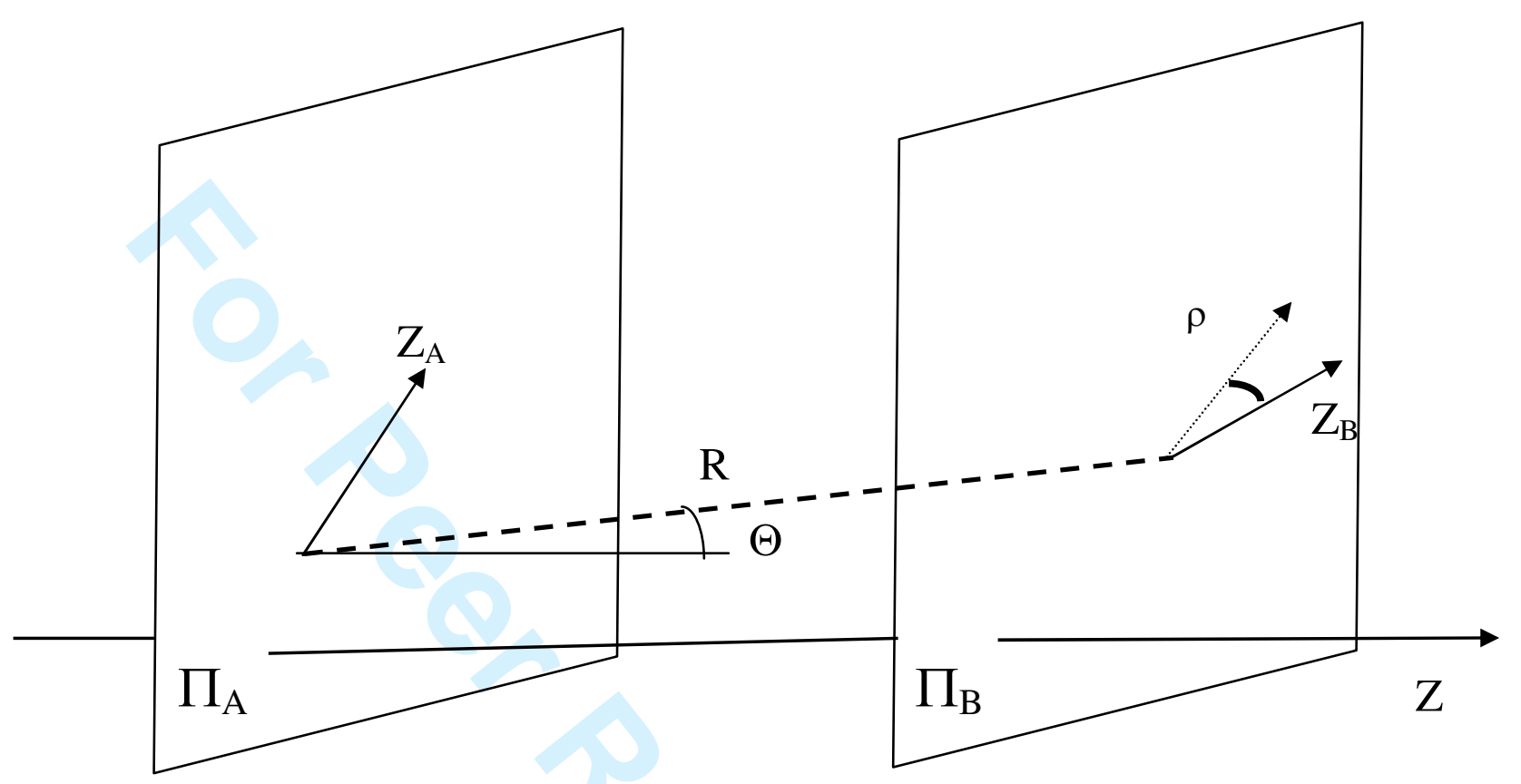

29

30

(31

34

35

36

37

38

39

40

41

42

43

44

45

46

47

48

49

50

51

52

53

54

55

56

57

58

59

60

URL: http://mc.manuscriptcentral.com/tandf/tmph 


\section{References:}

[1] L. Landau, E. Lifshitz, Electrodynamics of continuous media (MIR, Moscow, 1969)

[2] N. Bloembergen, Nonlinear optics (Benjamin, New-York, 1965)

[3] R. Bonneville, Molecular Physics, 106, 2627 (2008) and references therein

[4] P.E. Schipper, Chem. Phys. 26, 29 (1977)

[5] R.A. Harris, Mol. Phys. 105, 2433 (2007)

[6] R. Bonneville, Mol.Cryst.Liq.Cryst., Sci.Technol.-Sec.B : Nonlinear Optics, 2, 159

(1992)

[7] A.R. Edmonds, Angular momentum in quantum mechanics (Princeton University

Press, Princeton, 1960) 